\title{
Turduckening black holes: an analytical and computational study
}

\author{
David Brown, ${ }^{1}$ Peter Diener, ${ }^{2,3}$ Olivier Sarbach, ${ }^{4}$ Erik Schnetter, ${ }^{2,3}$ and Manuel Tiglio ${ }^{5,6}$ \\ ${ }^{1}$ Department of Physics, North Carolina State University, Raleigh, NC 27695, USA \\ ${ }^{2}$ Center for Computation \& Technology, Louisiana State University, Baton Rouge, LA 70803, USA \\ ${ }^{3}$ Department of Physics \& Astronomy, Louisiana State University, Baton Rouge, LA 70803, USA \\ ${ }^{4}$ Instituto de Física y Matemáticas, Universidad Michoacana de San Nicolás de Hidalgo, \\ Edificio C-3, Cd. Universitaria, C. P. 58040 Morelia, Michoacán, México \\ ${ }^{5}$ Center for Fundamental Physics, Department of Physics, \\ University of Maryland, College Park, MD 20742, USA \\ ${ }^{6}$ Center for Scientific Computation and Mathematical Modeling, \\ University of Maryland, College Park, MD 20742, USA
}

\begin{abstract}
We provide a detailed analysis of several aspects of the turduckening technique for evolving black holes. At the analytical level we study the constraint propagation for a general family of BSSN-type formulation of Einstein's field equations and identify under what conditions the turducken procedure is rigorously justified and under what conditions constraint violations will propagate to the outside of the black holes. We present high-resolution spherically symmetric studies which verify our analytical predictions. Then we present three-dimensional simulations of single distorted black holes using different variations of the turduckening method and also the puncture method. We study the effect that these different methods have on the coordinate conditions, constraint violations, and extracted gravitational waves. We find that the waves agree up to small but non-vanishing differences, caused by escaping superluminal gauge modes. These differences become smaller with increasing detector location.
\end{abstract}

PACS numbers: 04.20.-q,04.25.Dm,04.30.Db

\section{INTRODUCTION}

In a previous publication [1] we discussed the turduckening approach to numerical simulations of black holes in Einstein's theory. The technique relies on initially smoothing the data inside each black hole and solving the Einstein evolution equations everywhere at later times. The idea was first proposed in Ref. [2] under the name of "free black hole evolution". It shares many similarities with the "stuffed black hole" [3, 4] and "magic matter" [5] approaches. In Ref. [1] we presented a particular implementation that works in practice for binary black holes. We also provided justification for our implementation, and numerical evidence of the geometrical picture behind it. Complementary results based on very similar ideas were independently found and presented in Ref. [6] under the name "filling the holes".

The intuitive rationale behind the turduckening approach is that the physics in the exterior of a black hole should be causally disconnected from the unphysical smoothing in the interior. This is the same rationale behind black hole excision [7, 8], but here one proceeds in a different way. In particular, one does not need to place an inner boundary per black hole in order to remove the interior. The computational domain in this technique is trivial from a topological point of view, and therefore the discretization remains simple. Thus, the method shares the simplicity of the moving punctures technique [9, 10] but is not restricted to puncture-type initial data and does not require regularization of the equations near special points.

${ }^{*}$ URL: http://www.cct.lsu.edu/

${ }^{\dagger}$ URL: http://relativity.phys.lsu.edu/ 
In this paper we extend our analysis of the turducken technique, concentrating on both conceptual and practical issues.

We begin in Section [1 by describing the formulation of the equations that we used in Ref. [1], which is a specific version of the BSSN-type family. We analyze in detail the hyperbolicity of both the main system and the subsidiary (constraint) system, placing particular emphasis on the propagation speeds of constraint violating modes. It is well known that in the Einstein equations the "true" degrees of freedom are coupled to coordinate and constrained degrees of freedom. One therefore needs to guarantee that, for the formulation of the Einstein evolution equations and the gauge conditions being used, the smoothing in the interior of each black hole does not affect the "physics" in the exterior. This is a non-trivial condition and, in fact, it is formulation and gauge dependent. In Section II we show that there are some versions of the BSSN equations where this condition does not hold, and where constraint violations that originate in the interior of the black hole do propagate to the outside. However, we are also able to identify a class of BSSN-type equations for which we can rigorously guarantee that constraint violations inside the black hole do not leak to the outside.

Next we concentrate on the issue of whether gauge modes can escape from the interior of the black hole. The gauge conditions that we use are those of the moving punctures technique. In Section Пwe show that some of the characteristic speeds depend on the solution itself. Therefore it is not possible to determine a priori whether or not some modes will become superluminal. There is nothing wrong with modes leaking from the black hole interior, as long as these modes represent the gauge freedom inherent in the evolution problem. It is nevertheless of conceptual and practical importance to understand how the turduckening procedure might affect the gauge outside the black hole. Below we turn to this point by analyzing the numerical data.

Having analyzed the system of equations at the continuum, and in particular, having shown that at that level the turduckening procedure does not introduce constraint violations to the exterior of a black hole, we address the discretization and numerical implementation in the following sections. We begin in Sec. III with a brief description of the numerical codes that are used in this paper. In Sec. IV we evolve turduckened initial data for a Schwarzschild black hole with a spherically symmetric one-dimensional code. Using this code we can corroborate with high numerical accuracy that the constraint violations in the formulation of the equations that we use do not leak to the outside, as expected from our analytical analysis.

The one-dimensional numerical studies also reveal an interesting property of turducken evolutions: even though the stuffing procedure initially introduces large constraint violations inside the black holes, these violations quickly decay to very small values as the evolution proceeds. This occurs because the shift vector quickly moves the coordinate grid points away from the future domain of dependence of the turduckened region, while the constraint-violating modes are confined to the inside of the black hole. The numerical data then relax to a portion of the stationary $1+\log$ "trumpet slice" of the black hole [11, 12, 13]. This is the same end state as obtained with puncture evolution.

We also use one-dimensional simulations to investigate the possibility of superluminal gauge modes. We find that gauge modes are in fact superluminal and propagate from the interior to the exterior of the black hole. In particular, the smoothing procedure affects the coordinate conditions outside the black hole. However, we find that the differences in gauge that arise from different types of smoothing quickly decay in time. As already mentioned, we find that the turducken solution approaches a portion of the trumpet slice, regardless of the type of smoothing.

Given that it has already been shown in [1, [6] that the turduckening procedure works in practice for binary black hole evolutions, we next analyze in detail several aspects of single black hole evolutions. In Sec. $\mathrm{V}$ we present results from three-dimensional evolutions of a single distorted rotating black hole. This data is obtained by applying the smoothing procedure to puncture 
initial data with Bowen-York extrinsic curvature. We compare in detail the gauge conditions and extracted waveforms produced in calculations with turduckening regions of different sizes as well as a pure puncture evolution. We also show that we have fourth order convergence in the extracted waveforms.

We find, in agreement with the one-dimensional results, that superluminal gauge modes are able to propagate to the outside of the horizon. However, if the turduckening region is sufficiently small, the effect of these gauge modes decreases with radius outside of the black hole, and becomes small enough that for practical purposes it can be disregarded in 3D simulations.

Comparing the waveforms from turduckening and pure puncture runs, we find that the differences are very small and that most of them converge to zero with increasing resolution. The remaining differences are caused by the differences in gauge at the finite detector locations and are found to become smaller with increasing detector location.

Section VI contains some final remarks.

\section{FORMULATION OF THE EQUATIONS, CONSTRAINT PROPAGATION, HYPERBOLICITY, AND CHARACTERISTIC SPEEDS}

In this section we first give the explicit form of the evolution and constraint equations used in our Cauchy formulation of Einstein's field equations. It is a special case of the family of formulations analyzed in [14]. Next, we summarize the conditions under which this formulation is hyperbolic and give the characteristic speeds. Finally, we extend the analysis performed in [14] by deriving the constraint propagation system, describing the propagation of constraint violations, and analyzing its hyperbolic structure. In particular, we give necessary conditions for this system to be symmetric hyperbolic and possess no superluminal speeds. We then prove that under these conditions constraint violations inside a black hole which are present in the turducken approach cannot propagate to the domain of outer communication.

\section{A. Formulation of the equations}

As mentioned in the introduction, we consider a BSSN-type formulation of Einstein's equations where the three metric $\gamma_{i j}$ and the extrinsic curvature $K_{i j}$ are decomposed according to

$$
\begin{aligned}
\gamma_{i j} & =e^{4 \phi} \tilde{\gamma}_{i j}, \\
K_{i j} & =e^{4 \phi}\left(\tilde{A}_{i j}+\frac{1}{3} \tilde{\gamma}_{i j} K\right) .
\end{aligned}
$$

Here, the conformal factor $e^{2 \phi}$ is chosen such the conformal metric $\tilde{\gamma}_{i j}$ has unit determinant, and $K=\gamma^{i j} K_{i j}$ and $\tilde{A}_{i j}$ are the trace and the trace-less part, respectively, of the conformally rescaled extrinsic curvature. The $3+1$ decomposition of Einstein's equations along with suitable gauge conditions for lapse $(\alpha)$ and shift $\left(\beta^{i}\right)$ yields the following evolution system $[14]^{1}$

\footnotetext{
${ }^{1}$ There are two sign errors in Eqs. (5) and (6) of Ref. [14]. The first is in front of the second term of Eq. (5) and the second in front of the fourth term in Eq. (6). Since these errors only affect lower order terms they do not affect the results in [14] in any way. We thank Dae-Il Choi for pointing out these errors to us.
} 


$$
\begin{aligned}
\hat{\partial}_{0} \alpha= & -\alpha^{2} f\left(\alpha, \phi, x^{\mu}\right)\left(K-K_{0}\left(x^{\mu}\right)\right) \\
\hat{\partial}_{0} K= & -e^{-4 \phi}\left[\tilde{D}^{i} \tilde{D}_{i} \alpha+2 \partial_{i} \phi \cdot \tilde{D}^{i} \alpha\right]+\alpha\left(\tilde{A}^{i j} \tilde{A}_{i j}+\frac{1}{3} K^{2}\right)-\alpha S, \\
\hat{\partial}_{0} \beta^{i}= & \alpha^{2} G\left(\alpha, \phi, x^{\mu}\right) B^{i}, \\
\hat{\partial}_{0} B^{i}= & e^{-4 \phi} H\left(\alpha, \phi, x^{\mu}\right) \hat{\partial}_{0} \tilde{\Gamma}^{i}-\eta^{i}\left(B^{i}, \alpha, x^{\mu}\right) \\
\hat{\partial}_{0} \phi= & -\frac{\alpha}{6} K+\frac{1}{6} \partial_{k} \beta^{k} \\
\hat{\partial}_{0} \tilde{\gamma}_{i j}= & -2 \alpha \tilde{A}_{i j}+2 \tilde{\gamma}_{k(i} \partial_{j} \beta^{k}-\frac{2}{3} \tilde{\gamma}_{i j} \partial_{k} \beta^{k}, \\
\hat{\partial}_{0} \tilde{A}_{i j}= & e^{-4 \phi}\left[\alpha \tilde{R}_{i j}+\alpha R_{i j}^{\phi}-\tilde{D}_{i} \tilde{D}_{j} \alpha+4 \partial_{(i} \phi \cdot \tilde{D}_{j)} \alpha\right]^{T F} \\
& \left.+\alpha K \tilde{A}_{i j}-2 \alpha \tilde{A}_{i k} \tilde{A}_{j}^{k}+2 \tilde{A}_{k(i} \partial_{j}\right) \beta^{k}-\frac{2}{3} \tilde{A}_{i j} \partial_{k} \beta^{k}-\alpha e^{-4 \phi} \hat{S}_{i j}, \\
\hat{\partial}_{0} \tilde{\Gamma}^{i}= & \tilde{\gamma}^{k l} \partial_{k} \partial_{l} \beta^{i}+\frac{1}{3} \tilde{\gamma}^{i j} \partial_{j} \partial_{k} \beta^{k}+\partial_{k} \tilde{\gamma}^{k j} \cdot \partial_{j} \beta^{i}-\frac{2}{3} \partial_{k} \tilde{\gamma}^{k i} \cdot \partial_{j} \beta^{j} \\
& -2 \tilde{A}^{i j} \partial_{j} \alpha+2 \alpha\left[(m-1) \partial_{k} \tilde{A}^{k i}-\frac{2 m}{3} \tilde{D}^{i} K+m\left(\tilde{\Gamma}_{k l}^{i} \tilde{A}^{k l}+6 \tilde{A}^{i j} \partial_{j} \phi\right)\right]-S^{i},
\end{aligned}
$$

where we have introduced the operator $\hat{\partial}_{0}=\partial_{t}-\beta^{j} \partial_{j}$. Here, all quantities with a tilde refer to the conformal three metric $\tilde{\gamma}_{i j}$ and the latter is used in order to raise and lower indices. In particular, $\tilde{D}_{i}$ and $\tilde{\Gamma}_{i j}^{k}$ refer to the covariant derivative and the Christoffel symbols, respectively, with respect to $\tilde{\gamma}_{i j}$. The expression $[\cdots]^{T F}$ denotes the trace-less part (with respect to the metric $\tilde{\gamma}_{i j}$ ) of the expression inside the parentheses, and

$$
\begin{aligned}
& \tilde{R}_{i j}=-\frac{1}{2} \tilde{\gamma}^{k l} \partial_{k} \partial_{l} \tilde{\gamma}_{i j}+\tilde{\gamma}_{k(i} \partial_{j)} \tilde{\Gamma}^{k}-\tilde{\Gamma}_{(i j) k} \partial_{l} \tilde{\gamma}^{l k}+\tilde{\gamma}^{l s}\left(2 \tilde{\Gamma}_{l(i}^{k} \tilde{\Gamma}_{j) k s}+\tilde{\Gamma}_{i s}^{k} \tilde{\Gamma}_{k l j}\right), \\
& R_{i j}^{\phi}=-2 \tilde{D}_{i} \tilde{D}_{j} \phi-2 \tilde{\gamma}_{i j} \tilde{D}^{k} \tilde{D}_{k} \phi+4 \tilde{D}_{i} \phi \tilde{D}_{j} \phi-4 \tilde{\gamma}_{i j} \tilde{D}^{k} \phi \tilde{D}_{k} \phi .
\end{aligned}
$$

The gauge conditions imposed on the lapse, Eq. (3), is a generalization of the Bona-Massó condition [15] where $f\left(\alpha, \phi, x^{\mu}\right)$ is a smooth and strictly positive function and $K_{0}\left(x^{\mu}\right)$ is an arbitrary smooth function. The conditions imposed on the shift in Eqs. (566) is a generalization of the hyperbolic Gamma driver [16] condition where $G\left(\alpha, \phi, x^{\mu}\right)$ and $H\left(\alpha, \phi, x^{\mu}\right)$ are smooth, strictly positive functions, and $\eta^{i}\left(B^{j}, \alpha, x^{\mu}\right)$ is a smooth vector-valued function. The term $\hat{\partial}_{0} \tilde{\Gamma}^{i}$ in Eq. (6) is set equal to the right-hand side of Eq. (10). Note that we use the operator $\hat{\partial}_{0}$ (as opposed to $\partial_{t}$ ) in these gauge conditions; not only does this simplify the analysis of the principal part of the evolution equations, it also results in stable binary black hole evolutions for moving punctures [17] and the turducken approach [1].

Finally, the parameter $m$ which was introduced in [18], controls how the momentum constraint is added to the evolution equations for the variable $\tilde{\Gamma}^{i}$. The standard choice in numerical simulations is $m=1$ which eliminates the divergence of $\tilde{A}^{i j}$ in Eq. (10). However, we find it instructive not to fix $m=1$ in this article. The source terms $S, \hat{S}_{i j}$ and $S^{i}$ are defined in terms of the four Ricci tensor, $R_{i j}^{(4)}$, and the constraint variables

$$
\begin{aligned}
H & \equiv \frac{1}{2}\left(\gamma^{i j} R_{i j}^{(\gamma)}+K^{2}-K^{i j} K_{i j}\right), \\
M_{i} & \equiv \tilde{D}^{j} \tilde{A}_{i j}-\frac{2}{3} \tilde{D}_{i} K+6 \tilde{A}_{i j} \tilde{D}^{j} \phi \\
C_{\Gamma}^{i} & \equiv \tilde{\Gamma}^{i}+\partial_{j} \tilde{\gamma}^{i j}
\end{aligned}
$$


as

$$
\begin{aligned}
S & =\gamma^{i j} R_{i j}^{(4)}-2 H, \\
\hat{S}_{i j} & =\left[R_{i j}^{(4)}+\tilde{\gamma}_{k(i} \partial_{j} C_{\Gamma}^{k}\right]^{T F}, \\
S^{i} & =2 \alpha m \tilde{\gamma}^{i j} M_{j}-\hat{\partial}_{0} C_{\Gamma}^{i} .
\end{aligned}
$$

In vacuum, the evolution equations consist of Eqs. (3-10) with $S=0, \hat{S}_{i j}=0, S^{i}=0$. In order to obtain a solution to Einstein's vacuum field equations, one also has to solve the constraints $H=0$, $M_{i}=0$ and $C_{\Gamma}^{i}=0$. Below, we show that for $m=1$ it is sufficient to solve these constraints on an initial Cauchy surface in the region exterior to black holes. The constraint propagation system then guarantees that these constraints hold at all events which are future to the initial surface and outside the black hole regions, provided suitable boundary conditions are specified at the outer boundary of the computational domain.

\section{B. Hyperbolicity and characteristic speeds for the main system}

The evolution system (3-10) is first order in time and mixed first/second order in space. There exist at least three different methods for analyzing hyperbolicity (that is, the well-posedness of the Cauchy formulation) for such systems. The first method consists in reducing the system to fully first order by introducing extra variables (and constraints) and to show that the resulting first order system is strongly or symmetric hyperbolic (see [19] for definitions). The hyperbolicity of the BSSN equations with a fixed shift and a densitized lapse or a Bona-Massó type condition using this method has been established in Refs. [20] and [14]. The second method which was developed in Refs. 21, 22] is also based on a first order system. However, the reduction makes use of pseudo-differential operators. This has the advantage of not introducing any new constraints. Unlike the first method, this results in a unique first order system. The hyperbolicity of the BSSN equations with a Bona-Massó type condition and a hyperbolic Gamma driver type condition was shown in [14] using this method. Finally, the third method which has been proposed in Ref. [23] and applied to BSSN in Ref. [24] consists in finding an energy norm which, in the limit of frozen coefficients, is conserved. This method has been shown [25] to be equivalent to obtaining a first order symmetric hyperbolic reduction with the first method.

Based on the second method, the following characteristic speeds with respect to normal observers for the evolution system (3,[10) were found in [14]: $0, \pm \mu_{1}, \pm \mu_{2}, \pm \mu_{3}, \pm \mu_{4}, \pm \mu_{5}$ and $\pm \mu_{6}$ where

$$
\begin{gathered}
\mu_{1}=\sqrt{f}, \quad \mu_{2}=\sqrt{\frac{4 m-1}{3}}, \quad \mu_{3}=\sqrt{m}, \\
\mu_{4}=1, \quad \mu_{5}=\sqrt{G H}, \quad \mu_{6}=\sqrt{\frac{4 G H}{3} .}
\end{gathered}
$$

When considering high-frequency perturbations of smooth solutions it is possible to classify the characteristic fields as gauge fields, constraint-violating fields and gravitational radiation [26, 27]. According to this classification, the fields propagating with speeds $\mu_{1}, \mu_{5}$ and $\mu_{6}$ correspond to gauge modes, the fields propagating with speeds $\mu_{2}$ and $\mu_{3}$ to constraint-violating modes and the fields propagating with speeds $\mu_{4}$ to gravitational radiation. As shown below, this statement can be strengthened by noticing that $0, \mu_{2}$ and $\mu_{3}$ are the characteristic speeds of the constraint propagation system. In fact, it can be shown 28] under quite general assumptions that the characteristic speeds of the constraint propagation system are a subset of the speeds of the main evolution system.

In 14] the following necessary conditions for strong hyperbolicity are given: $f>0, m>1 / 4$ and $G H>0$ or $f>0, m>1 / 4$ and $G=H=0$. (Notice that for $G=H=0$ the evolution equation 
for the shift, Eq. (5), decouples from the remaining system.) If, in addition, the parameter $m$ and the functions $f, G$ and $H$ can be chosen such that the functions

$$
\frac{4 G H}{3 f-4 G H}, \quad \frac{6(m-1)}{4 m-1-4 G H}, \quad \frac{2(m-1) G H}{m-G H}
$$

have smooth limits at $3 f=4 G H, 4 m=1+G H$ and $m=G H$, then strong hyperbolicity is guaranteed [14]. For the standard choice $m=1$ it is sufficient to verify that $f>0, G H>0$ and that the function $4 G H /(3 f-4 G H)$ has a smooth limit at $3 f=4 G H$.

In the three-dimensional simulations below, we fix the functions $f, K_{0}$ and $G, H$ and $\eta^{i}$ as follows. We choose the $1+\log$ condition

$$
f=\frac{2}{\alpha}, \quad K_{0}=0
$$

and the Gamma-driver shift condition

$$
G=\frac{3}{4 \alpha^{2}}, \quad H=e^{4 \phi}, \quad \eta^{i}=\eta B^{i}
$$

with $\eta=1 / 2$. In this case, $\mu_{1}=\sqrt{2 / \alpha}$ and strong hyperbolicity is guaranteed if the function $2 \alpha e^{-4 \phi}-1$ does not cross zero. In our initial slices $\alpha \rightarrow 1$ and $\phi \rightarrow 0$ in the asymptotic region while near black holes $\alpha>0$ is small ( $\alpha \approx 0.3$ at the horizon) and $\phi$ positive. Therefore there already exists a two-surface where the condition $2 \alpha e^{-4 \phi}-1 \neq 0$ is violated in the initial data. On the other hand, since this surface is a set of zero measure in the computational domain there is hope that the violation of our sufficient conditions at this surface might still result in a well posed Cauchy problem. The numerical simulations below show no apparent sign of instability.

\section{Hyperbolicity and characteristic speeds of the constraint propagation system}

Next, we derive the constraint propagation system which describes the propagation of constraint violations. We prove that for $1 / 4<m \leq 1$ constraint violations inside a black hole region cannot propagate to the outside.

A convenient way of finding the constraint propagation system is to perform a $3+1$ decomposition of the contracted Bianchi identities, $2 \nabla^{\mu} R_{\mu \nu}^{(4)}-\nabla_{\nu} R^{(4)}=0$, where one sets the quantities $S, \hat{S}_{i j}$ and $S^{i}$ defined in Eqs. (16-18) to zero. Taking into account the definitions of the constraint variables $H, M_{j}$ and $C_{\Gamma}^{i}$ defined in Eqs. (13)-15) one finds that they obey the linear evolution system

$$
\begin{aligned}
\hat{\partial}_{0} H & =-\frac{1}{\alpha} D^{j}\left(\alpha^{2} M_{j}\right)-\alpha e^{-4 \phi} \tilde{A}^{i j} \tilde{\gamma}_{k i} \partial_{j} C_{\Gamma}^{k}+\frac{2 \alpha}{3} K H, \\
\hat{\partial}_{0} M_{j} & =\frac{\alpha^{3}}{3} D_{j}\left(\alpha^{-2} H\right)+\alpha K M_{j}+M_{i} \partial_{j} \beta^{i}+D^{i}\left(\alpha\left[\tilde{\gamma}_{k(i} \partial_{j} C_{\Gamma}^{k}\right]^{T F}\right), \\
\hat{\partial}_{0} C_{\Gamma}^{i} & =2 \alpha m \tilde{\gamma}^{i j} M_{j} .
\end{aligned}
$$

In order to analyze this system, which is mixed first/second order in space, we use the first method described in Sec. IIB and reduce it to a first order symmetric hyperbolic system. This allows us to establish the causal propagation of the constraints via a standard energy inequality. Introducing the additional constraint variable $Z_{i}^{k}=\partial_{i} C_{\Gamma}^{k}, Z_{i j}=Z_{i}{ }^{k} \tilde{\gamma}_{k j}$, Eqs. (21)23) can be rewritten as the following first order linear system: 


$$
\begin{aligned}
\hat{\partial}_{0} H & =-\frac{1}{\alpha} D^{j}\left(\alpha^{2} M_{j}\right)-\alpha e^{-4 \phi} \tilde{A}^{i j} Z_{i j}+\frac{2 \alpha}{3} K H \\
\hat{\partial}_{0} M_{j} & =\frac{\alpha^{3}}{3} D_{j}\left(\alpha^{-2} H\right)+\alpha K M_{j}+M_{i} \partial_{j} \beta^{i}+D^{i}\left(\alpha Z_{(i j)}\right)^{T F}-\sigma \alpha e^{-4 \phi}\left[\partial_{k} Z_{j}^{k}-\partial_{j} Z_{k}^{k}\right], \\
\hat{\partial}_{0} C_{\Gamma}^{i} & =2 \alpha m \tilde{\gamma}^{i j} M_{j} . \\
\hat{\partial}_{0} Z_{i j} & =2 m \partial_{i}\left(\alpha M_{j}\right)-2 \alpha m \tilde{\gamma}^{k l}\left(\partial_{i} \tilde{\gamma}_{j k}\right) M_{l}-2 \alpha \tilde{A}_{k j} Z_{i}^{k} \\
& +Z_{i k} \partial_{j} \beta^{k}+Z_{k j} \partial_{i} \beta^{k}+Z_{i}^{k} \tilde{\gamma}_{l j} \partial_{k} \beta^{l}-\frac{2}{3} Z_{i j} \partial_{k} \beta^{k} .
\end{aligned}
$$

Here, we have included in the right-hand side of Eq. (25) the term $\partial_{k} Z_{j}{ }^{k}-\partial_{j} Z_{k}{ }^{k}$ with an arbitrary factor $\sigma$. Since $Z_{i}^{k}=\partial_{i} C_{\Gamma}^{k}$, this term is identically zero. However, as we will see now, its addition allows greater flexibility in obtaining a symmetric hyperbolic system. The system (24)|27) has the form

$$
\hat{\partial}_{0} C=\alpha\left[\mathbf{A}(u)^{i} \partial_{i} C+\mathbf{B}(u) C\right],
$$

where $C$ are the constraint variables, $u=\left(\alpha, \beta^{i}, \phi, K, \tilde{\gamma}_{i j}, \tilde{A}_{i j}\right)$ are the main variables, and $\mathbf{A}^{i}$, $i=1,2,3$, and $\mathbf{B}$ are matrix-valued functions of $u$. Decomposing $Z_{i j}=\hat{Z}_{(i j)}+Z_{[i j]}+\gamma_{i j} Z / 3$ into its trace-free symmetric part, $\hat{Z}_{(i j)}$, its antisymmetric part, $Z_{[i j]}$, and its trace, $Z=\gamma^{i j} Z_{i j}=$ $e^{-4 \phi} Z_{k}{ }^{k}$, and representing $C$ in terms of the variables $C=\left(C_{\Gamma}^{i}, S_{1}:=2 m H+Z, S_{2}:=H+\right.$ $\left.2 \sigma Z, M_{j}, \hat{Z}_{(i j)}, Z_{[i j]}\right)$, the principal symbol $\mathbf{A}(\mathbf{n})=\mathbf{A}(u)^{i} n_{i}$ is given by

$$
\mathbf{A}(\mathbf{n})\left(\begin{array}{c}
C_{\Gamma}^{i} \\
S_{1} \\
S_{2} \\
M_{j} \\
\hat{Z}_{(i j)} \\
\left.Z_{[i j]}\right)
\end{array}\right)=\left(\begin{array}{c}
0 \\
0 \\
(4 m \sigma-1) n^{j} M_{j} \\
\frac{1}{3} n_{j} S_{2}+(1-\sigma) n^{i} \hat{Z}_{(i j)}+\sigma n^{i} Z_{[i j]} \\
2 m\left(n_{(i} M_{j)}\right)^{T F} \\
2 m n_{[i} M_{j]}
\end{array}\right) .
$$

Here $n^{i} \equiv \gamma^{i j} n_{j}$ and $n_{i}$ is normalized such that $n_{i} n^{i}=1$. This system is symmetric hyperbolic if and only if the following inequalities hold:

$$
4 m \sigma-1>0, \quad 2 m(1-\sigma)>0, \quad 2 m \sigma>0,
$$

which is equivalent to the two conditions $m>1 / 4$ and $1 /(4 m)<\sigma<1$. Therefore, as long as $m>1 / 4$ (which is also a necessary condition for the main evolution system to be strongly hyperbolic, see Sec. IIB) we can choose $\sigma$ between $1 /(4 m)$ and 1 and obtain a symmetric hyperbolic constraint propagation system. For the standard choice $m=1$, for instance, we can choose $\sigma=1 / 2$ which is the case considered in [1]. A symmetrizer $\mathbf{H}=\mathbf{H}^{T}$ is given by

$$
\begin{aligned}
C^{T} \mathbf{H} C & =\tilde{\gamma}_{i j} C_{\Gamma}^{i} C_{\Gamma}^{j}+S_{1}^{2}+\frac{1}{3(4 m \sigma-1)} S_{2}^{2}+\gamma^{i j} M_{i} M_{j} \\
& +\frac{1-\sigma}{2 m} \gamma^{i k} \gamma^{j l} \hat{Z}_{(i j)} \hat{Z}_{(k l)}+\frac{\sigma}{2 m} \gamma^{i k} \gamma^{j l} Z_{[i j]} Z_{[k l]} .
\end{aligned}
$$

$\mathbf{H}$ is positive definite and satisfies $\mathbf{H A}(\mathbf{n})=\mathbf{A}(\mathbf{n})^{T} \mathbf{H}$. The symmetrizer allows us to obtain an energy-type estimate $^{2}$ for the constraint variables $C$. For this, define the four-current

$$
J^{\mu} \partial_{\mu}=\frac{1}{2 \alpha} C^{T} \mathbf{H} C \hat{\partial}_{0}-\frac{1}{2} C^{T} \mathbf{H} \mathbf{A}^{i} C \partial_{i} .
$$

\footnotetext{
${ }^{2}$ Such estimates are a standard technique in the theory of hyperbolic partial differential equations. In particular, they allow one to prove uniqueness and continuous dependence on the data and to establish the principle of finite propagation speed. For references, see for instance [28, 29].
} 
By virtue of Eq. (28) the current satisfies the conservation law

$$
\partial_{\mu} J^{\mu} \equiv \partial_{t} J^{t}+\partial_{i} J^{i}=C^{T} \mathbf{L} C,
$$

with $2 \mathbf{L}=\mathbf{H B}+(\mathbf{H B})^{T}+\partial_{t}\left(\alpha^{-1} \mathbf{H}\right)-\partial_{i}\left(\mathbf{H A}^{i}+\alpha^{-1} \beta^{i} \mathbf{H}\right)$. Next, let $\Omega_{T}=\bigcup_{0 \leq t \leq T} \Sigma_{t}$ be a tubular region obtained by piling up open subsets $\Sigma_{t}$ of $t=$ const hypersurfaces. This region is enclosed by the space-like hypersurfaces $\Sigma_{0}, \Sigma_{T}$ and the surface $\mathcal{T}:=\bigcup_{0 \leq t \leq T} \partial \Sigma_{t}$, which is assumed to be smooth. Integrating (30) over $\Omega_{T}$ and using Gauss' theorem in $\mathbb{R}^{4}$ with the Euclidean metric, one obtains

$$
\int_{\Sigma_{T}} J^{t} d^{3} x=\int_{\Sigma_{0}} J^{t} d^{3} x+\int_{\Omega} C^{T} \mathbf{L} C d^{4} x-\int_{\mathcal{T}} J^{\mu} e_{\mu} d S,
$$

where $e_{\mu}$ is the outward unit one-form normal to $\mathcal{T}$ and $d S$ the volume element on that surface. If the boundary term $J^{\mu} e_{\mu}$ is positive or zero, one obtains the estimate

$$
\begin{aligned}
\int_{\Sigma_{T}} J^{t} d^{3} x & \leq \int_{\Sigma_{0}} J^{t} d^{3} x+\int_{\Omega} C^{T} \mathbf{L} C d^{4} x \\
& =\int_{\Sigma_{0}} J^{t} d^{3} x+\int_{0}^{T}\left(\int_{\Sigma_{t}} C^{T} \mathbf{L} C d^{3} x\right) d t \\
& \leq \int_{\Sigma_{0}} J^{t} d^{3} x+b \int_{0}^{T}\left(\int_{\Sigma_{t}} J^{t} d^{3} x\right) d t,
\end{aligned}
$$

where $b$ is a constant and where we have used the positivity of $J^{t}=C^{T} \mathbf{H} C /(2 \alpha)$ in the last step. By Gronwall's lemma, one obtains the inequality

$$
\int_{\Sigma_{t}} J^{t} d^{3} x \leq e^{b t} \int_{\Sigma_{0}} J^{t} d^{3} x, \quad 0 \leq t \leq T .
$$

Since $2 \alpha J^{t}=C^{T} \mathbf{H} C$ is positive definite, this then implies that $C=0$ everywhere on $\Omega_{T}$ if $C=0$ on $\Sigma_{0}$ which shows that it is sufficient to solve the constraints $C=0$ on the initial slice $\Sigma_{0}$. In view of numerical applications, however, the constraints are not exactly satisfied on $\Sigma_{0}$. Instead, numerical errors introduced by solving the constraint equations on a finite grid may be modeled by a sequence $C_{n}$ of initial constraint violations which converges to zero as the resolution goes to infinity. In this case, the estimate (31) shows that for each fixed $t \in[0, T]$ the $L^{2}$-norm of the constraint variables $C_{n}$ converge to zero on $\Sigma_{t}$.

In order to analyze the conditions under which the boundary term is nonnegative it is convenient to expand the outward normal vector as $e_{\mu} d x^{\mu}=N\left[a \alpha d t+n_{i}\left(d x^{i}+\beta^{i} d t\right)\right]$ where $n_{i}$ is normalized such that $\gamma^{i j} n_{i} n_{j}=1$ and $N>0$ is a normalization factor. We set $N=1$ in the following since we are only interested in the sign of the boundary term. Notice that $|a|<1$ if $\mathcal{T}$ is time-like, $|a|>1$ if $\mathcal{T}$ is space-like and $|a|=1$ if $\mathcal{T}$ is a null surface. With this notation, the boundary term is equal to

$$
J^{\mu} e_{\mu}=\frac{1}{2} C^{T}\left[a \mathbf{H}-\mathbf{H A}^{i} n_{i}\right] C .
$$

Therefore, the condition for this boundary term to be positive or zero is that all the eigenvalues of the matrix $\mathbf{A}^{i} n_{i}$ are smaller than or equal to $a$. If $\mathcal{T}$ is a future event horizon, then $a=1$ and this 
condition means that all of the eigenvalues must be smaller than or equal to one. For the symbol given in Eq. (29) these eigenvalues are the characteristic speeds (with respect to normal observers) and are

$$
0, \quad \pm \mu_{2}= \pm \sqrt{\frac{4 m-1}{3}}, \quad \pm \mu_{3}= \pm \sqrt{m}
$$

In particular, there are no superluminal speeds if $1 / 4<m \leq 1$, and in this case no constraint violations can propagate out of a black hole. For the standard choice $m=1$ this condition is satisfied. However, we also see that choosing $m>1$ in the evolution equation for $\tilde{\Gamma}^{i}$, Eq. (10), yields superluminal constraint speeds in which case constraint violations inside a black hole can affect the exterior region.

Finally, we would like to point out that if the computational domain contains time-like boundaries, then $|a|<1$ and the sign of the boundary term $J^{\mu} e_{\mu}$ is not automatically positive or zero. In this case, boundary conditions need to be specified such that this term is nonnegative and such that a well posed Cauchy problem is obtained.

\section{Spherical symmetry}

The BSSN equations can be specialized to spherical symmetry as described in Ref. [30]. The first step is to remove the restriction $\tilde{\gamma}=1$ on the determinant of the conformal metric and replace it with an evolution equation for $\tilde{\gamma}$ [31]. In this paper we use the "Eulerian evolution" defined by $\partial_{t} \tilde{\gamma}=2 \tilde{\gamma} \tilde{D}_{i} \beta^{i}$. The conformal connection functions are defined in terms of the conformal Christoffel symbols by $\tilde{\Gamma}^{i} \equiv \tilde{\gamma}^{j k} \tilde{\Gamma}_{j k}^{i}$.

The reduction to spherical symmetry is achieved by writing the conformal metric as $d s^{2}=$ $\tilde{\gamma}_{r r} d r^{2}+\tilde{\gamma}_{\theta \theta} d^{2} \Omega$, where $d^{2} \Omega$ is the metric for the unit two-sphere. The independent component of the trace-free part of the extrinsic curvature is $\tilde{A}_{r r}$, and the independent component of the conformal connection functions is $\tilde{\Gamma}^{r}$.

In one dimension as in three, we use $1+\log$ slicing and the Gamma-driver shift condition (although here we use $\eta=1$ for the damping parameter). For the main evolution system in spherical symmetry the characteristic speeds are $0, \pm \mu_{1}, \pm \mu_{2}$ and $\pm \mu_{5}$. Strong hyperbolicity is guaranteed for $f>0, m>1 / 4$ and $G H>0$ if the following conditions hold: $f \neq G H$ and $3 G H+1 \neq 4 m$.

The constraint evolution system can be obtained by spherical reduction of the system (21, 23), or by direct calculation from the $1 \mathrm{D}$ equations of motion [30]. Let $Z_{r}^{r} \equiv \partial_{r} C_{\Gamma}^{r}$ and define the vector of constraints by $C \equiv\left(H, M_{r}, C_{\Gamma}^{r}, Z_{r}^{r}\right)^{T}$. The constraint evolution equations have the form $\hat{\partial}_{0} C=\alpha\left[\mathbf{A}^{r} \partial_{r} C+\mathbf{B} C\right]$ where $\mathbf{A}^{r}$ and $\mathbf{B}$ are functions of the BSSN variables. The principal symbol is given by

$$
\mathbf{A}^{r}=\left(\begin{array}{cccc}
0 & -2 e^{-4 \phi} / \tilde{\gamma}_{r r} & 0 & 0 \\
1 / 6 & 0 & 0 & 2 e^{-4 \phi} / 3 \\
0 & 0 & 0 & 0 \\
0 & 2 m / \tilde{\gamma}_{r r} & 0 & 0
\end{array}\right)
$$

The characteristic fields are $C_{\Gamma}^{r}, m H+e^{-4 \phi} Z_{r}^{r}$, and $H \pm 2 \sqrt{3(4 m-1) / \tilde{\gamma}_{r r}} e^{-2 \phi} M_{r}+4 e^{-4 \phi} Z_{r}^{r}$ with proper speeds 0,0 , and $\pm \mu_{2}= \pm \sqrt{(4 m-1) / 3}$, respectively. This system is symmetric hyperbolic as long as $4 m>1$. For the case of primary interest, $m=1$, the characteristic fields propagate along the normal and along the light cone.

A symmetrizer can be constructed from the squares of the characteristic fields: 


$$
\begin{aligned}
C^{T} \mathbf{H C}= & \left(m H+e^{-4 \phi} Z_{r}^{r}\right)^{2}+\left(C_{\Gamma}^{r}\right)^{2}+\left(H+2 \sqrt{3(4 m-1) / \tilde{\gamma}_{r r}} e^{-2 \phi} M_{r}+4 e^{-4 \phi} Z_{r}^{r}\right)^{2} \\
& +\left(H-2 \sqrt{3(4 m-1) / \tilde{\gamma}_{r r}} e^{-2 \phi} M_{r}+4 e^{-4 \phi} Z_{r}^{r}\right)^{2} \\
= & \left(m^{2}+2\right) H^{2}+\left(C_{\Gamma}^{r}\right)^{2}+24(4 m-1) e^{-4 \phi}\left(M_{r}\right)^{2} / \tilde{\gamma}_{r r} \\
& +33 e^{-8 \phi}\left(Z_{r}^{r}\right)^{2}+2 e^{-4 \phi}(m+8) H Z_{r}^{r} .
\end{aligned}
$$

The spacetime current, defined by

$$
J^{\mu} \partial_{\mu}=\frac{1}{2 \alpha} C^{T} \mathbf{H} C \hat{\partial}_{0}-\frac{1}{2} C^{T} \mathbf{H} \mathbf{A}^{r} C \partial_{r},
$$

satisfies the conservation law

$$
\partial_{\mu} J^{\mu} \equiv \partial_{t} J^{t}+\partial_{r} J^{r}=C^{T} \mathbf{L} C
$$

where $2 \mathbf{L}=\mathbf{H B}+\mathbf{B}^{T} \mathbf{H}+\partial_{t}(\mathbf{H} / \alpha)-\partial_{r}\left(\mathbf{H}\left(\mathbf{A}^{r}+\beta^{r} / \alpha\right)\right)$. As in the $3 \mathrm{D}$ case, we can now show that if $J^{\mu} e_{\mu}$ is non-negative at the boundaries, then

$$
\int_{\Sigma_{t}} J^{t} d r \leq e^{b t} \int_{\Sigma_{0}} J^{t} d r, \quad 0 \leq t \leq T
$$

for some constant $b$. It follows that the constraints will vanish on $\Sigma_{t}$ if they vanish on the initial hypersurface $\Sigma_{0}$.

The assumption that $J^{\mu} e_{\mu}$ is non-negative at the boundaries for $m=1$ can be seen to hold at the black hole horizon by following the same reasoning as in the three-dimensional case. We expand the Euclidean normal one form $e_{\mu}$ as a linear combination of the unit normal $u_{\mu}$ to the $t=$ const surfaces and the unit normal $n_{i}$ to the two-dimensional boundary within the spacelike hypersurfaces. As in the three-dimensional case, we have $e_{\mu} d x^{\mu}=N\left[a \alpha d t+n_{r}\left(d x^{r}+\beta^{r} d t\right)\right]$ where $|a|=1$ characterizes a null surface. By dropping the positive constant $N$, we find $J^{\mu} e_{\mu}=C^{T}\left(a \mathbf{H}-\mathbf{H} \mathbf{A}^{r} n_{r}\right) C / 2$. This shows that for a black hole horizon $J^{\mu} e_{\mu}$ is positive if the eigenvalues of $\mathbf{A}^{r} n_{r}$ are less than or equal to one. This is indeed the case for $m=1$, since the constraint propagation system has eigenvalues 0 and $\pm \sqrt{(4 m-1) / 3}$.

\section{CODE DESCRIPTIONS}

We use two different codes for the simulations presented in this paper. One of them is McLachlan, a three-dimensional adaptive mesh refinement code, which uses the BSSN system of equations, as described in [16, 32], with the gauge conditions described in section \above. See section II for the exact form of these equations. The McLachlan code is a Cactus thorn which is entirely generated by Kranc [33, 34, 35] directly from equations and differencing stencils specified in Mathematica format. McLachlan uses the Cactus framework [36, 37] and the Carpet mesh refinement driver [38, 39]. The evolution code is fully fourth order accurate in time and space. We use fifth order spatial interpolation at mesh refinement boundaries, using buffer zones as described in [38] to ensure stability and convergence at mesh refinement boundaries, and using tapered grids as described in [40] to avoid the Berger-Oliger time interpolation. Our finite differencing operators are the standard centered fourth order accurate first and second finite differencing operators, except for the advection terms which are upwinded (also fourth order). We use a fourth order Runge-Kutta time integrator and add fifth order Kreiss-Oliger dissipation [41] to the right hand sides. We apply standard radiation (Sommerfeld) boundary conditions (as described in [16]) to 
all components of the evolved fields. These boundary conditions are neither 4th order convergent, nor constraint preserving so non-convergent constraint violations will propagate inwards from the outer boundary. We therefore expect our code, in the limit of infinite resolution, to be fully fourth order accurate only in the region that is causally disconnected from the outer boundaries. We place our outer boundaries far enough out that they do not affect our wave extraction procedure.

We center a stack of refined regions around the origin. Each region is a cube with a resolution half of the next coarser region.

We also use a one-dimensional code to analyze several issues related to the turducken technique in the context of a spherically symmetric black hole. This is the code used in [12] and described in detail as the "Eulerian case" in [30]. The 1D code uses a uniform radial grid with nodes at coordinate radii $r_{j}=(j-1 / 2) \Delta r$, where $j=1,2$, etc. Fourth order finite differencing is used for spatial derivatives, and fourth order Runge-Kutta is used for the time update. No boundary conditions are imposed at the origin for any of the variables except the shift vector component $\beta^{r}$. That is, for all variables except $\beta^{r}$, the finite difference stencil is shifted toward positive $r$ near the origin so that no guard cells are needed. For $\beta^{r}$ we impose the boundary condition $\partial_{r} \beta^{r}=0$ at $r=0$ by using a fourth order, one sided finite difference representation of $\partial_{r} \beta^{r}$. The resulting guard cell value is

$$
\beta^{r}(0)=\frac{1}{22}\left[17 \beta^{r}(1)+9 \beta^{r}(2)-5 \beta^{r}(3)+\beta^{r}(4)\right]
$$

where the numbers in parentheses label grid points. In the evolution code, spatial derivatives of $\beta^{r}$ are computed by shifting the finite difference stencil toward positive $r$ near the origin. In this way only the single guard cell value $\beta^{r}(0)$ is needed. Numerical experiments show that the condition (37) (or a similar one) is needed for stability.

The one-dimensional code uses the variable $\chi \equiv e^{-4 \phi}$ rather than $\phi$. The CFL (CourantFriedrichs-Lewy) factor is 0.25 , and unless otherwise stated the resolution for all the onedimensional runs discussed here is $\Delta r=M / 200$.

\section{SINGLE BLACK HOLE EVOLUTIONS AND THE END STATE}

In this section we investigate the effects of black hole smoothing on the evolution of a spherically symmetric single black hole.

We start with the (Schwarzschild) isotropic black hole data

$$
\begin{aligned}
\tilde{\gamma}_{r r} & =1 \\
\tilde{\gamma}_{\theta \theta} & =r^{2} \\
e^{\phi} & =1+M /(2 r) \\
\tilde{\Gamma}^{r} & =-2 / r
\end{aligned}
$$

along with $K=0$ and $\tilde{A}_{r r}=0$. For all one-dimensional simulations, we use the $1+\log$ slicing and Gamma driver shift conditions as described in Sec. IIB. The initial values for the gauge variables are $\alpha=1$ and $\beta^{r}=B^{r}=0$. Unless otherwise stated, all simulations use $m=1$.

The black hole interior is turduckened by making the replacement $r \rightarrow \bar{r}(r)$ in the data (38) for $0 \leq r \leq r_{t}$, where

$$
\bar{r}(r) \equiv r_{0}-\left(10 r_{0}-4 r_{t}\right) r^{2} / r_{t}^{2}+\left(20 r_{0}-6 r_{t}\right) r^{3} / r_{t}^{3}-\left(15 r_{0}-4 r_{t}\right) r^{4} / r_{t}^{4}+\left(4 r_{0}-r_{t}\right) r^{5} / r_{t}^{5}
$$


The function $\bar{r}$ has the properties $\bar{r}(0)=r_{0}, \bar{r}^{\prime}(0)=0, \bar{r}\left(r_{t}\right)=r_{t}, \bar{r}^{\prime}\left(r_{t}\right)=1, \bar{r}^{\prime \prime}\left(r_{t}\right)=0$, and $\bar{r}^{\prime \prime \prime}\left(r_{t}\right)=0$. Thus, with this turduckening, we extend $r$ inside $r_{t}$ in such a way that the initial data are $C^{3}$ at $r_{t}$ and nonsingular at $r=0$. The lack of smoothness at $r_{t}$ generates an error in any centered fourth order finite difference derivative whose stencil extends across $r_{t}$. For first derivatives the error is $\mathcal{O}\left(\Delta r^{3}\right)$, and for second derivatives the error is $\mathcal{O}\left(\Delta r^{2}\right)$. Note that initially the horizon is located at coordinate radius $r=M / 2$. As long as $r_{t}<M / 2$, the turduckening lies entirely inside the black hole.

The turducken runs considered below use either $r_{0}=0.1 M, r_{t}=0.4 M$ or $r_{0}=0.05 M, r_{t}=$ $0.45 \mathrm{M}$. These two types of smoothing are referred to as case TA and case TB, respectively. We compare these results to results obtained with puncture data, denoted $\mathrm{P}$, which is equivalent to no turduckening (puncture evolution).

\section{A. Behavior of constraint violations}

Initially the constraint violations are restricted to the black hole interior, and our analysis in Section II shows that for $m \leq 1$ they should stay there. Thus, we expect the constraints to hold everywhere and at all times in the black hole exterior.

One obvious way to test this is to monitor the numerical constraints as functions of time, and confirm that the violations introduced by the turduckening do not leak out of the black hole. This is indeed the case. For smoothing types TA and TB, the initial Hamiltonian constraint violation inside the black hole is $\sim 10^{-1} / M^{2}$, while (at resolution $\Delta r=M / 200$ ) the initial constraint violation outside the black hole is $\sim 10^{-9} / M^{2}$. The constraint violation outside the black hole remains $\sim 10^{-9} / M^{2}$ throughout the evolution.

Moreover, we find that the region of constraint violation quickly shrinks relative to the numerical grid, and the constraints quickly loose memory of the turduckening. This comes about because the grid points surrounding the origin acquire a radially outward velocity that becomes superluminal within a time of a few $M$. The curves labeled "coord" in Figs. 1 show the proper speed of the coordinates in the radial direction with respect to observers at rest in the spacelike hypersurfaces. The speed is plotted as a function of proper distance from the black hole horizon, with the convention that positive values are outside the black hole and negative values are inside the black hole. What we see from these figures is that the coordinate grid inside the black hole moves faster than the speed of light $(c=1)$ in the radially outward direction. The region of constraint violation moves causally, with speeds 0 and \pm 1 . Thus, the coordinates soon move outside the future light cone of the stuffed region, and into the forward light cone of the initial data that satisfies the constraints. The graphs in Fig. 1 were taken from simulations with smoothing type TA. The graphs obtained with smoothing TB are nearly identical.

In Figs. 2 we plot the Hamiltonian constraint as a function of coordinate radius for the initial data $t=0$ and for the later times $t=4 M, 8 M$, and $12 M$. The data for turduckening types TA and TB are shown, as well as for puncture data $\mathrm{P}$. The constraint violation does not propagate beyond the black hole horizon. The value of the Hamiltonian constraint violation inside the black hole drops from $\sim 0.1$ to $\sim 10^{-9}$ by $t=12 M$. Beyond about $t=12 M$, the constraint data for TA and TB are everywhere (that is, also inside the black hole) indistinguishable from each other, and indistinguishable from the results obtained with puncture data $\mathrm{P}$.

The success of the turduckening procedure depends on the constraint violating modes propagating with speeds less than or equal to one. Our analysis in Section Mpredicts that this condition is met for the BSSN family of evolution equations (3)-(10) if and only if $1 / 4<m \leq 1$. In Fig. 3 we plot the numerical Hamiltonian constraint as a function of coordinate radius $r$ for $m=1.25$. One can clearly see that, as predicted by the theory, in this case the constraint violation does 


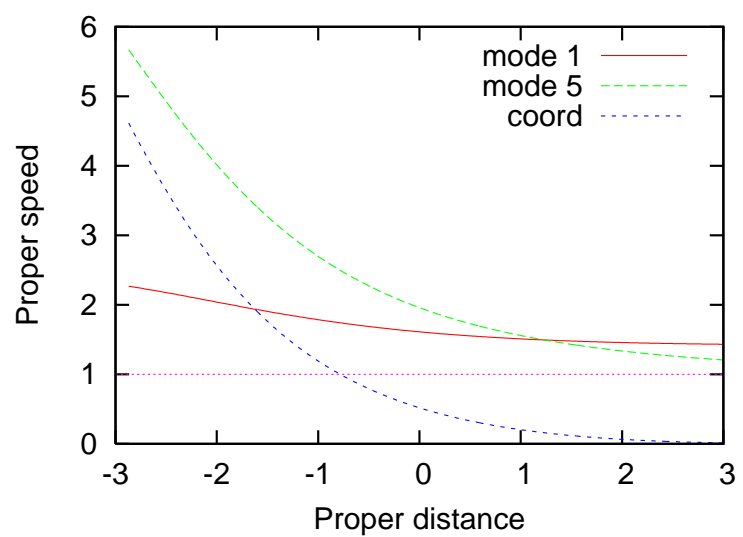

(a) Time $t=3 M$.

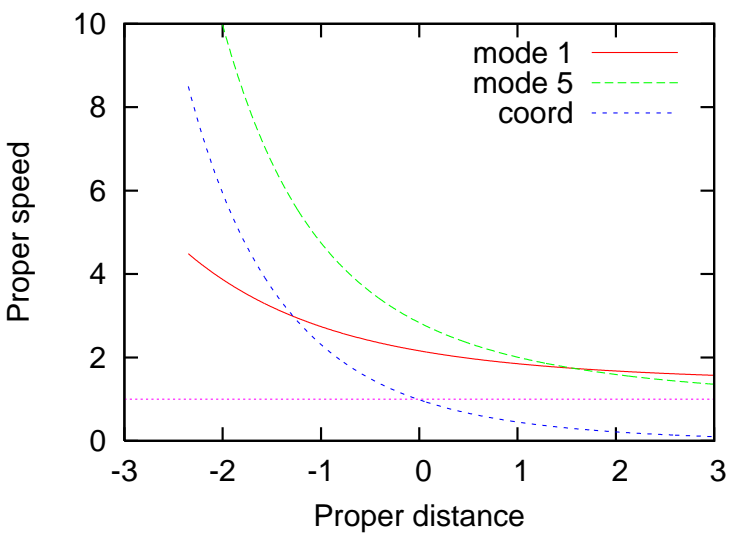

(b) Time $t=6 M$.

Figure 1: Proper speeds as a function of proper distance from the horizon for the turduckening case TA. The curve labeled 'coord' is the proper speed of the coordinate system with respect to the normal observers, $\sqrt{\tilde{\gamma}_{r r}} e^{2 \phi} \beta^{r} / \alpha$. The curve labeled "mode 1" is the proper speed $\mu_{1}=\sqrt{2 / \alpha}$. The curve labeled "mode 5" is the proper speed $\mu_{5}=\sqrt{3} e^{2 \phi} /(2 \alpha)$. The horizontal line is light speed.

propagate from the interior to the exterior of the black hole. This illustrates the fact that for a given formulation of the Einstein equations one cannot simply assume that the constraints will propagate with non-superluminal speeds.

\section{B. Behavior of the coordinates}

The relatively large value of the radial component of the shift vector moves the grid points beyond the region of constraint violation within a time of a few $M$. However, the grid points do not move beyond the influence of the turduckening. Recall that the main evolution system in spherical symmetry is strongly hyperbolic with characteristic speeds $0, \pm \mu_{1}= \pm \sqrt{2 / \alpha}, \pm \mu_{2}= \pm 1$, and $\pm \mu_{5}= \pm \sqrt{3} e^{2 \phi} /(2 \alpha)$. The modes with speeds $\mu_{1}$ and $\mu_{5}$ can become superluminal. Figure 1 shows the speeds $\mu_{1}$ and $\mu_{5}$ at times $t=3 M$ and $t=6 M$, along with the speed of the coordinate grid relative to the normal observers. Both speeds $\mu_{1}$ and $\mu_{5}$ are larger than the coordinate speed at the black hole horizon (the origin of proper distance). As discussed in Sec. IIB in some sense the modes corresponding to $\mu_{1}$ and $\mu_{5}$ can be associated with gauge freedom. We therefore expect that the turduckening process can affect gauge conditions outside the black hole. Although the full Einstein equations are satisfied in the black hole exterior, independent of the smoothing, the slicing and coordinate conditions outside the black hole can depend on the details of the stuffing.

Figure 4 shows the differences between lapse functions versus areal radius $R$, at four different times. For the curve TA-P, we compute the difference between the lapse function for smoothing TA and the lapse function obtained from puncture evolution (no smoothing). These calculations require some explanation. The areal radius is not a monotonic function of the coordinate radius; rather, the areal radius has a minimum at the black hole throat. Therefore for each of the runs TA and $\mathrm{P}$ we use only the data for which $R$ is an increasing function of coordinate radius. We then consider the overlap region in which $R$ is increasing for both data sets TA and P. The difference in lapse values is computed by interpolating this data onto a common grid that covers the overlap region. The differences $\mathrm{TB}-\mathrm{P}$ are computed in the same way, but with data from smoothing type TB. Note that the overlap regions extend inside the black hole horizon, which has areal radius $R=2 M$. 


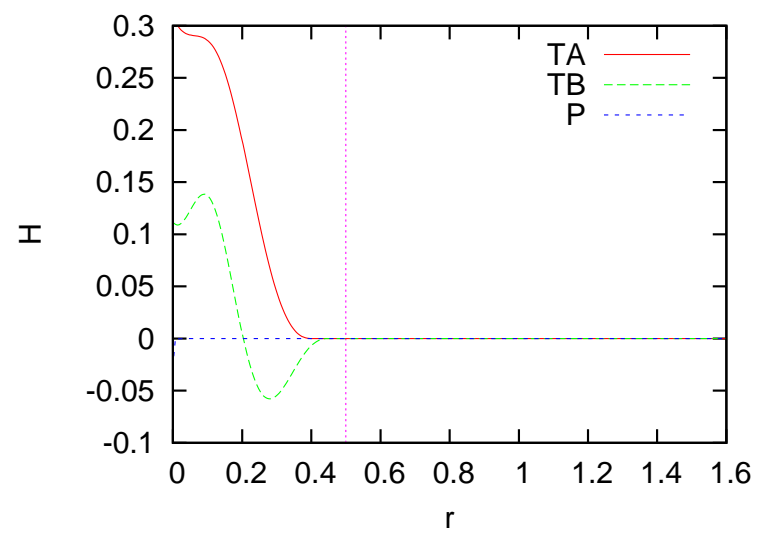

(a) Time $t=0 M$. The horizon location is $r=0.5 M$. At this scale the curve $\mathrm{P}$ cannot be distinguished from the horizontal axis.

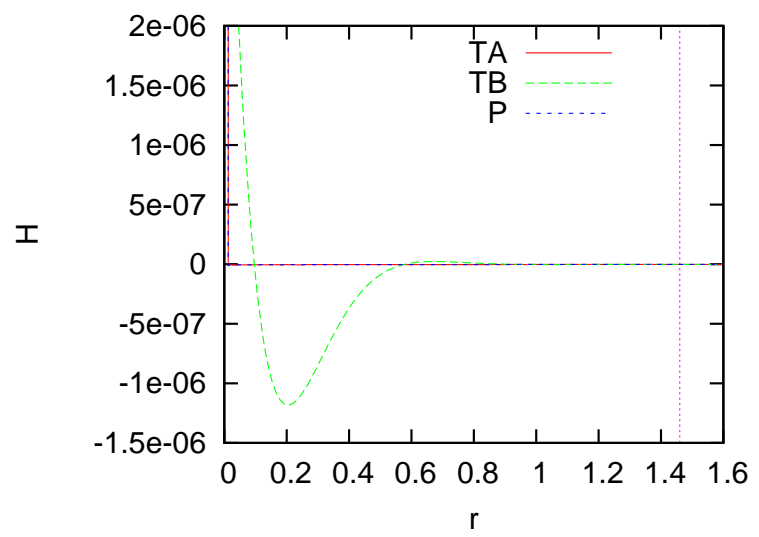

(c) Time $t=8 M$. The horizon location is $r \approx 1.46 M$. At this scale the curves TA and $\mathrm{P}$ cannot be distinguished from the horizontal axis.

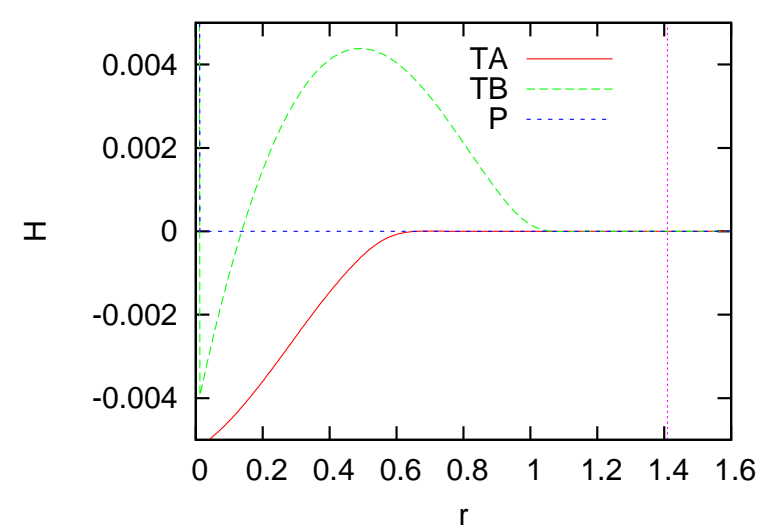

(b)Time $t=4 M$. The horizon location is $r \approx 1.41 M$.

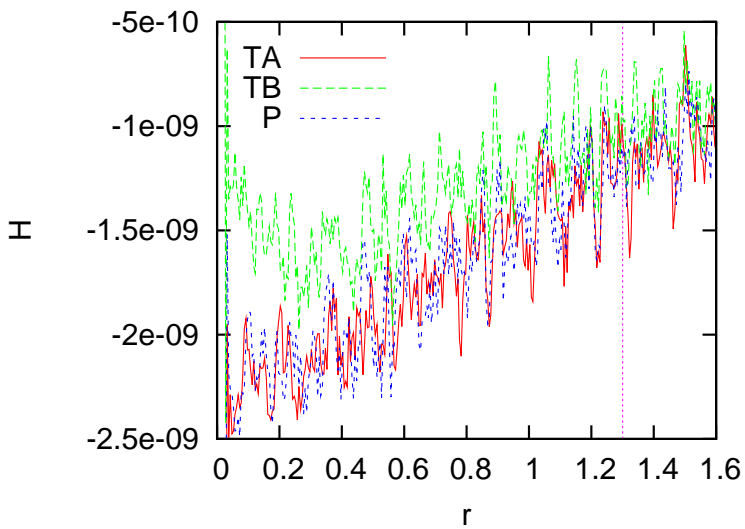

(d) Time $t=12 M$. The horizon location is $r \approx 1.30 M$. Curves TA and $\mathrm{P}$ are virtually identical. Curve TB shows some small differences that disappear shortly after $t=12 M$.

Figure 2: Hamiltonian constraint versus coordinate radius $r$ at various times, in units of $M$. TA and TB denote two types of turduckening, and $\mathrm{P}$ is puncture data. The vertical line shows the location of the black hole horizon.

The effect that the turduckening has on the slicing is relatively small when compared to the nominal value of 1 for the lapse, but is clearly seen in Fig. 4. This effect begins inside the black hole and propagates superluminally to the outside, where it continues to spread radially outward.

The turduckening's effect on the slicing condition fades with time. As the evolution proceeds, the data relax to a portion of a stationary $1+\log$ slice of a Schwarzschild black hole, independent of the initial stuffing details. This stationary $1+\log$ slice has a "trumpet" geometry [13]. It is the same final slice obtained with puncture evolution [12, 42, 43]. In Fig. 5] we graph the areal radius as a function of proper distance from the horizon (with the convention that positive distances are outside the black hole, negative distances are inside). These plots show the data for smoothing types TA and TB, and for no smoothing $\mathrm{P}$, at early $(t=0.25 M)$ and late $(t=50 M)$ times. The stationary $1+\log$ slice of Schwarzschild is shown as the curve labeled S. Initially the $R$ versus proper distance relation shows a strong dependence on smoothing. By $t=50 M$ all of the data $\mathrm{TA}, \mathrm{TB}$, and $\mathrm{P}$ have relaxed to approximate a portion of the trumpet slice $\mathrm{S}$. At this time, and with resolution $\Delta r=M / 200$, the numerical slices all end at proper distance $\approx-6.45 M$. Close 


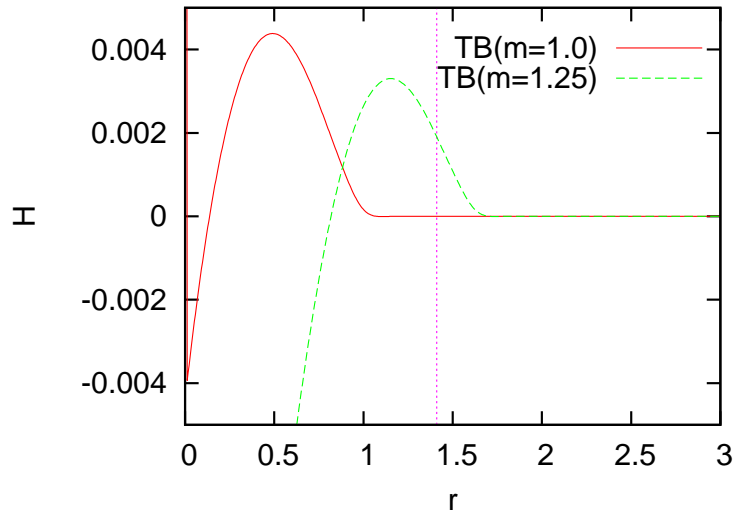

(a) Time $t=4 M$. With $m=1.25$, the constraint violation is beginning to spread to the black hole exterior.

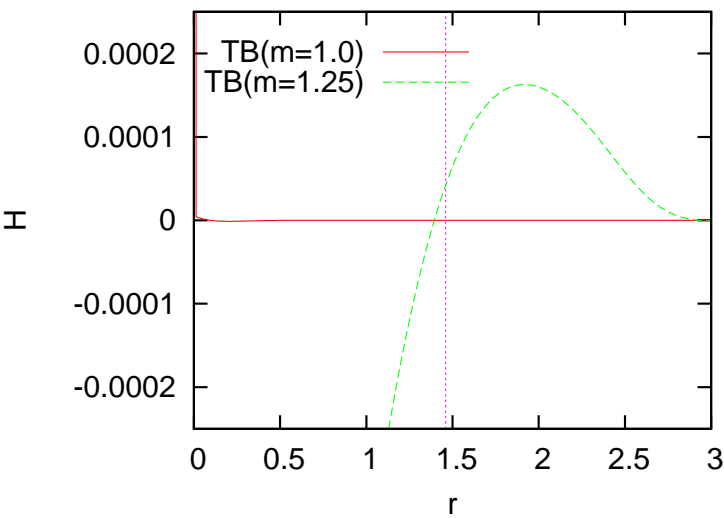

(b) Time $t=8 M$. With $m=1.25$ a pulse of constraint violating data has moved beyond the black hole and continues to propagate outward.

Figure 3: Hamiltonian constraint versus coordinate radius $r$ for $m=1$ and $m=1.25$, where $m$ is the parameter that controls the mixing of the momentum constraint in the equation of motion for $\Gamma^{r}$. The smoothing type TB is used in both cases. The vertical line shows the location of the black hole horizon. As predicted by the theory, the constraint violations inside the black hole do propagate to the outside if $m>1$.

inspection of the data shows that near the end of the numerical slice, the areal radius for the cases TA, TB, and $\mathrm{P}$ agree with one another to more than seven decimal places; at proper distance $-6 M$, we find $\alpha \approx 1.32013$. The areal radius for a stationary $1+\log$ slice at proper distance $-6 M$ is $\alpha \approx 1.32018$.

Although the stuffing can affect the slicing beyond the black hole horizon, it does not always do so. For the type of simulations here considered, it appears that any stuffing that is initially inside a coordinate radius of about $0.2 M$ remains causally disconnected from the black hole exterior. In Fig. [ 6 we show the difference between lapse functions for stuffing types TD, TE and puncture data $\mathrm{P}$. The cases TD and TE use turduckening radii $r_{t}=0.2 M$ and $r_{t}=0.3 M$, respectively. Both cases use $r_{0}=0.02 M$. The figure shows the common logarithm of the lapse differences at $t=3 \mathrm{M}$ and $t=6 M$, for low $(\Delta r=M / 100)$ and high $(\Delta r=M / 200)$ resolutions.

The top two subfigures show that the lapse difference TD-P converges to zero with increasing resolution. On the log plots the difference between low and high resolution curves is $\log (16) \approx 1.2$. These results show that with its small turduckening radius, the data for stuffing type TD are indistinguishable from puncture data in the black hole exterior. The bottom two subfigures show the lapse difference TE-P. In this case the difference converges to a nonzero value which spreads from the black hole interior to its exterior.

Let us refer once again to Fig. 1, which shows the gauge propagation speeds $\mu_{1}, \mu_{5}$ along with the coordinate system speed. Observe that the speed $\mu_{1}=\sqrt{f}$ depends on the slicing condition (3)), while the speed $\mu_{5}=\sqrt{G H}$ depends on the coordinate shift conditions (5) and (6). Thus it is the speed $\mu_{1}$ that we examine closely here. Figure 1 shows that the coordinate system moves faster than $\mu_{1}$ within a proper distance of $\sim M$ of the puncture. This appears to be a common result, independent of the stuffing details. If the stuffing initially extends beyond this $\mu_{1}$-sphere, where the speed curve for $\mu_{1}$ crosses the coordinate speed curve, then the stuffing's affect on the slicing can propagate radially outward through the black hole horizon. This is the case for data type TE. If the stuffing is initially contained entirely within the $\mu_{1}$-sphere, then the stuffing's affect on the slicing is lost as the coordinate grid quickly moves beyond the influence of mode $\mu_{1}$. This is the case for data type TD. 


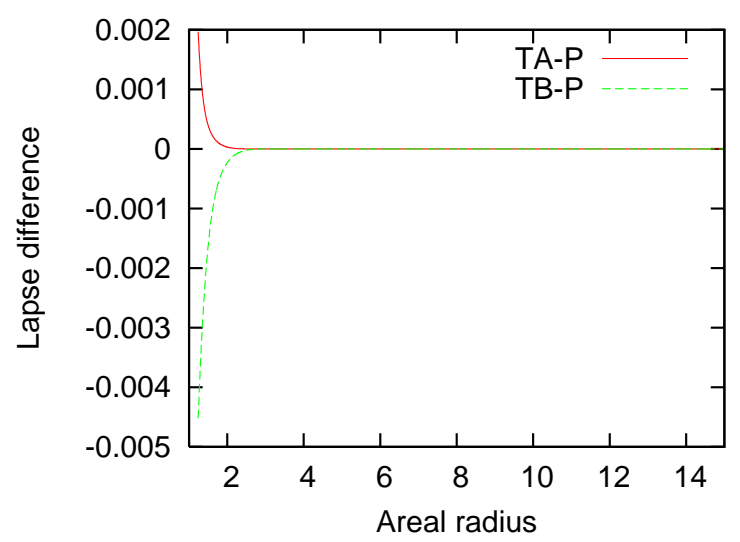

(a) Time $t=4 M$.

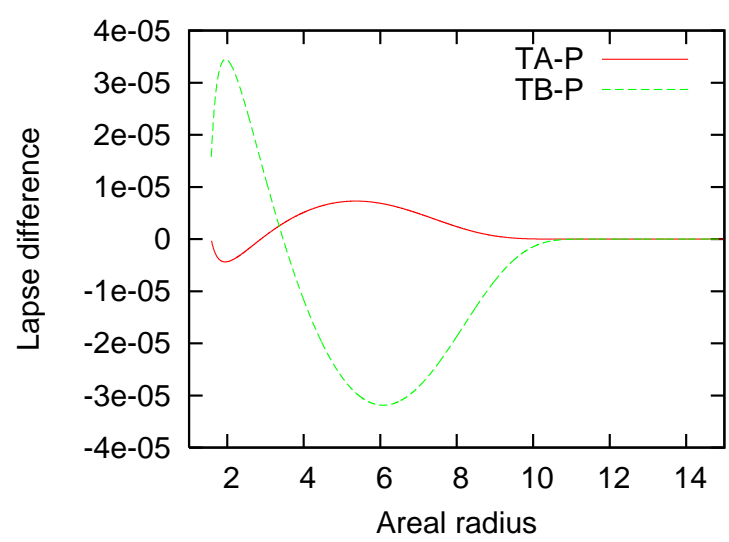

(c) Time $t=12 M$.

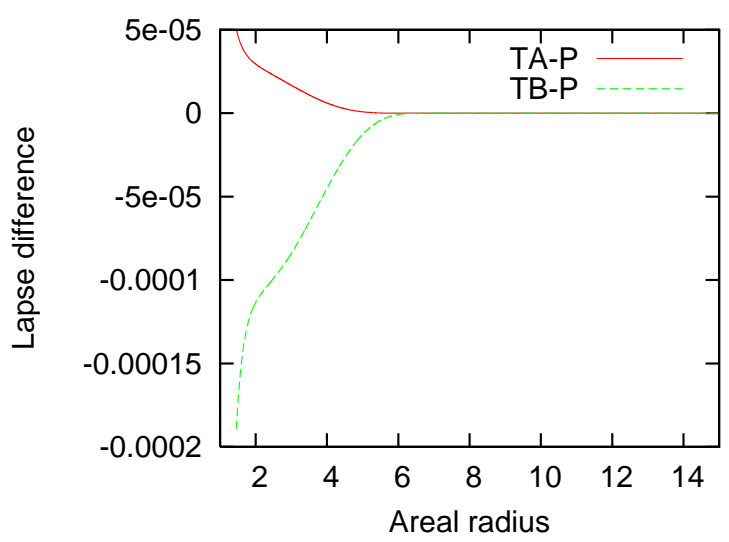

(b) Time $t=8 M$.

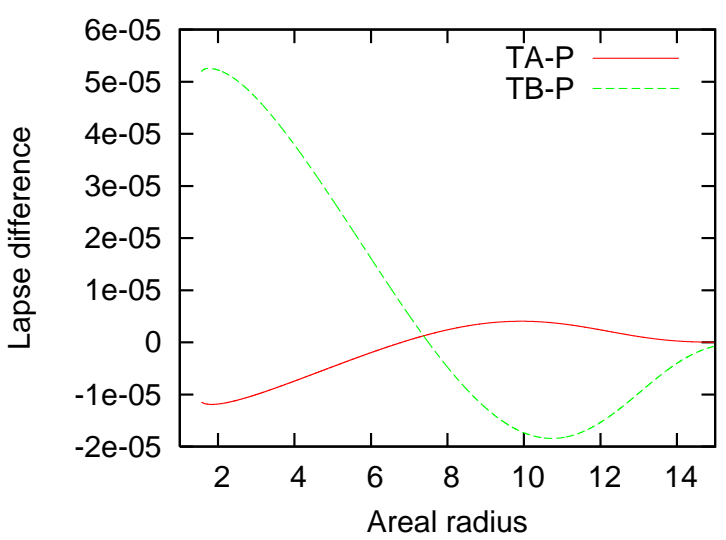

(d)Time $t=16 M$.

Figure 4: The difference between lapse functions plotted versus areal radius in units of $M$. The curve TA-P is the difference for turducken data with stuffing TA and puncture data. The curve TB-P is the difference for turducken data with stuffing TB and puncture data.

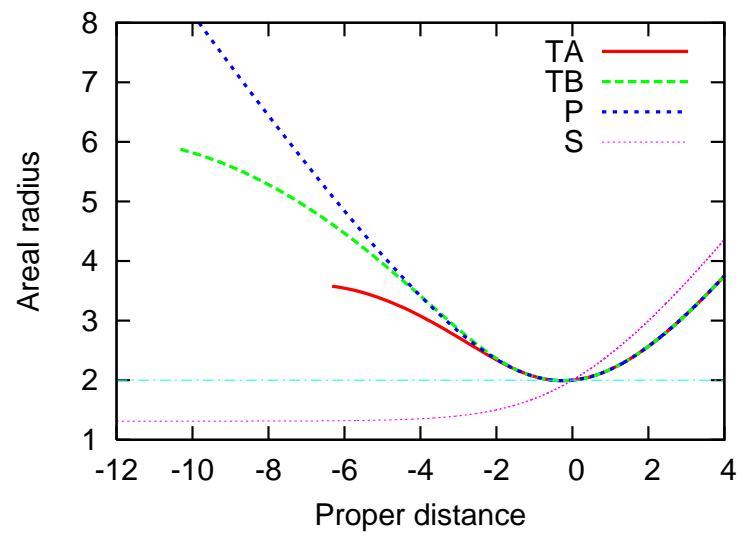

(a)Areal radius versus proper distance from the horizon at $t=M / 4$.

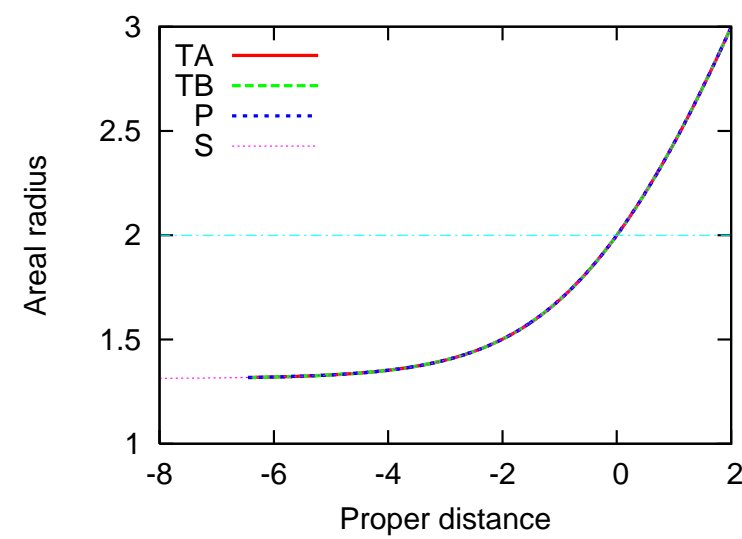

(b) Areal radius versus proper distance from the horizon at $t=50 \mathrm{M}$.

Figure 5: Areal radius $R$ versus proper distance from the horizon. The horizontal line at $R=2$ is the horizon. By $t=50 M$ the three curves (TA, TB, and $\mathrm{P}$ ) are indistinguishable (at this scale) from the stationary $1+\log$ slice $\mathrm{S}$. 


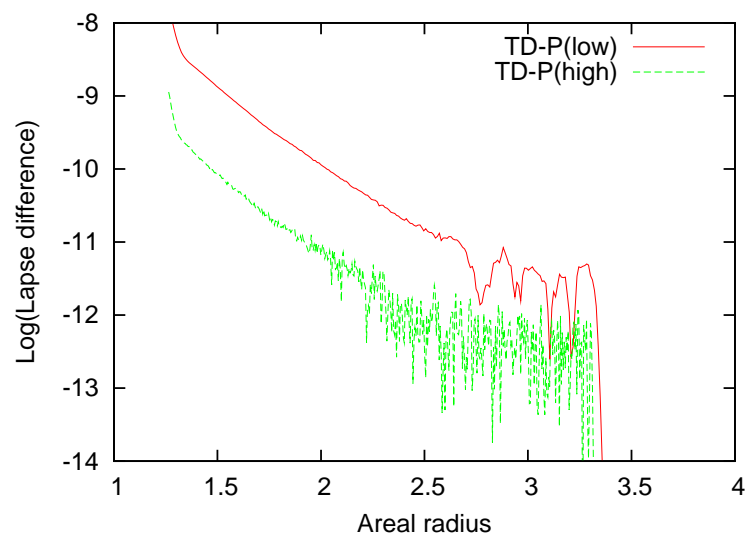

(a) Time $t=3 M$, turducken radius $r_{t}=0.2$.

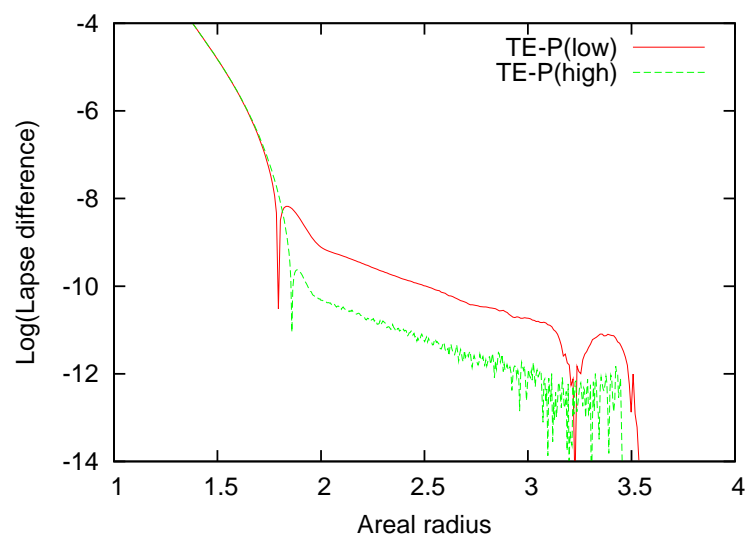

(c) Time $t=3 M$, turducken radius $r_{t}=0.3$.

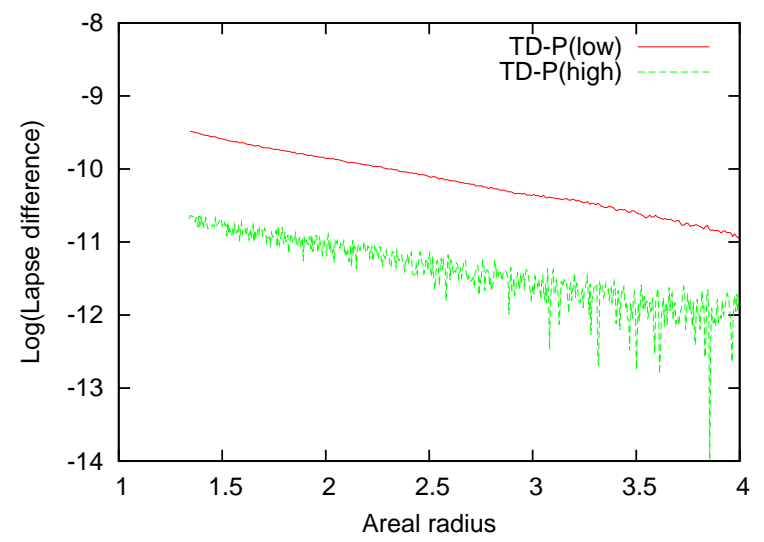

(b) Time $t=6 M$, turducken radius $r_{t}=0.2$.

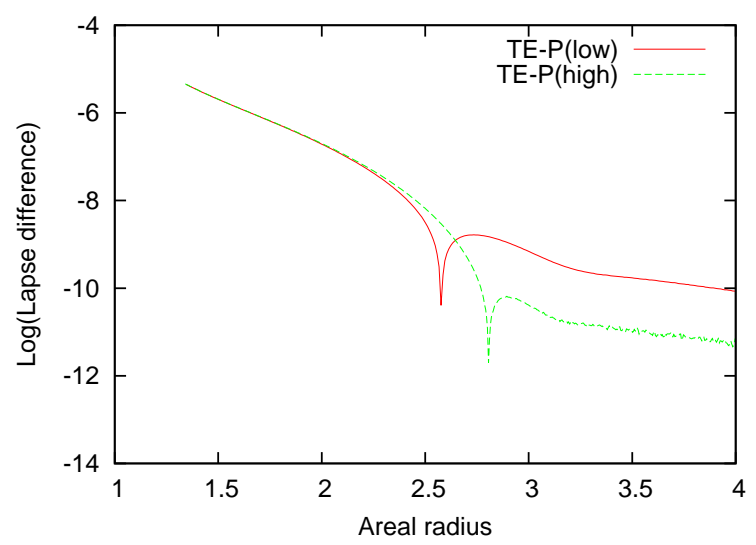

(d) Time $t=6 M$, turducken radius $r_{t}=0.3$.

Figure 6: Common logarithm of the difference between lapse functions. The top two subfigures show the difference between case TD and puncture data for low $(\Delta r=M / 100)$ and high $(\Delta r=M / 200)$ resolutions. The bottom two subfigures show the difference between case TE and puncture data for low and high resolutions. (Note that the curves at time $t=3 M$ appear to terminate at an areal radius of about 3.3 (case TD) or 3.5 (case TE). This occurs because, beyond these values, the lapse difference is exactly 0.0 to thirteen or more decimal places. The logarithm is undefined for larger values of areal radius.)

Note that the precise value of the turduckening radius where the influence of the gauge speed $\mu_{1}$ is lost might depend on a number of details of the simulation. For example, black hole spin, the initial values of the lapse and shift, as well as the details of the stuffing profile might affect whether or not the gauge modes will be lost in the black hole interior.

Finally, let us observe that the gauge speed $\mu_{5}$ is larger than the coordinate speed throughout the black hole interior. ${ }^{3}$ Thus, we expect that the stuffing details will affect the shift in the black hole exterior, regardless of how small the stuffing region might be. This expectation is supported by the results shown in Fig. 7 This figure shows the difference in coordinate radius for turducken data TD and puncture data $\mathrm{P}$ as a function of areal radius. As discussed above, in the limit of infinite resolution the slicing outside the black hole appears to be identical for turducken data TD and puncture data $\mathrm{P}$. Thus the difference shown in Fig. 7 is due to differences in the coordinate grid. In particular, the radial shift in the coordinate grid in the black hole exterior depends on the

\footnotetext{
${ }^{3}$ This observation partially explains why the one-dimensional code [30] seems to require boundary conditions at the origin for the shift vector.
} 
type of stuffing used. At time $t=2 M$ the difference has just begun to cross the black hole horizon. The difference continues to move into the black hole exterior as the evolution proceeds.

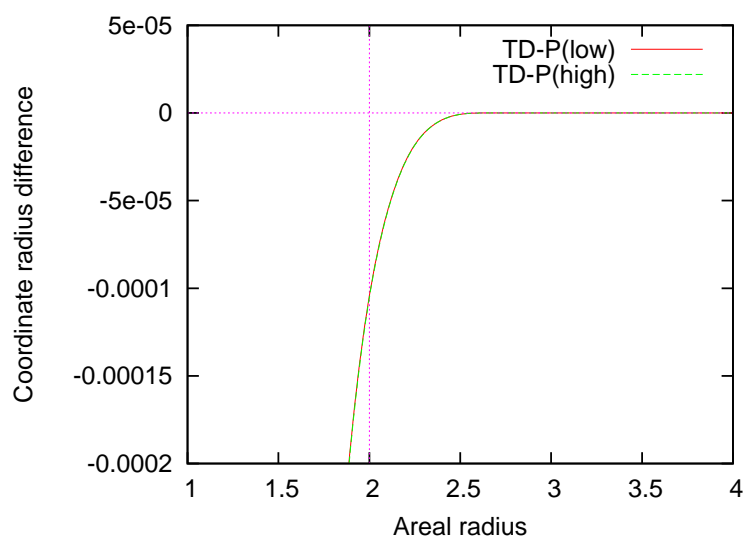

(a) Time $t=2 M$.

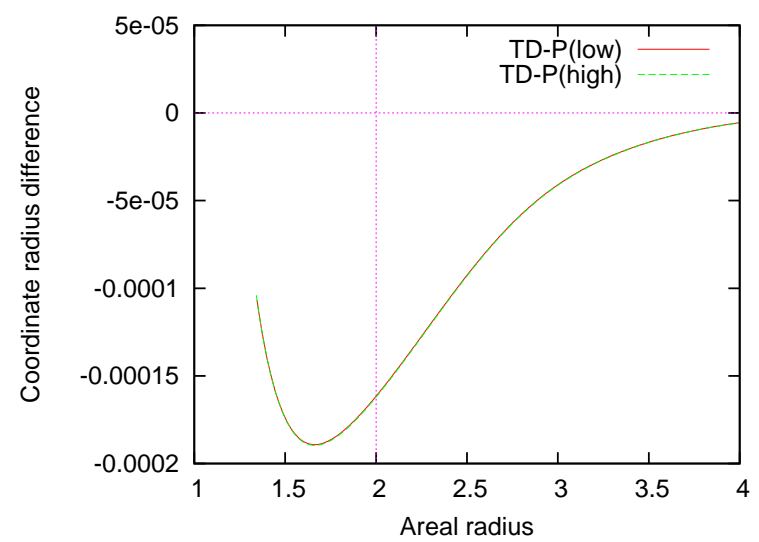

(b) Time $t=6 M$.

Figure 7: The difference between coordinate radius for turducken data TD and puncture data, as a function of areal radius. Both low $(\Delta r=M / 100)$ and high $(\Delta r=M / 200)$ resolution results are shown, although the curves are difficult to distinguish. The vertical line is the location of the black hole horizon.

\section{THREE-DIMENSIONAL BLACK HOLE EVOLUTIONS}

\section{A. Turduckening procedure in $3 \mathrm{D}$}

Numerical initial data with black holes may contain singular "puncture" points or excised regions. If the data are incomplete due to excision, the excised regions must be filled before the turducken method can be used to evolve the data. Even if the data are complete, as in the puncture case, it may be advantageous to replace the data near singular points with data that is more smooth.

We experimented with various methods for turduckening the initial data in the black hole interior. We found that the details do not matter much in practice, as long as the fields are sufficiently smooth across the turduckening boundary and the spacetime remains unchanged within a layer inside the horizon whose width is at least a few times the width of the finite differencing stencil. Empirically, a width of 10 grid points suffices for us; we expect this number to depend on the particular differencing operators which are used.

One rather simple method for turduckening is blending, which fills the excised region with arbitrary data, and then modifies some of the non-excised grid points to create a smooth match. This has the disadvantage that it may require quite a few non-excised grid points inside the horizon - one needs to have sufficiently many grid points to ensure the smooth match, plus the grid points which need to be left unmodified.

Instead, we choose a method which leaves all given data unchanged and fills in all excised points in a smooth manner. In particular, we solve an elliptic equation of the form

$$
\left(\frac{\partial^{n}}{\partial x^{n}}+\frac{\partial^{n}}{\partial y^{n}}+\frac{\partial^{n}}{\partial z^{n}}\right) A=0
$$

to fill the excised points of a quantity $A$, using standard centered derivatives everywhere and using the given non-excised data as boundary conditions. Here $n$ is an even integer which controls the 


\begin{tabular}{lc|rrr} 
& $\begin{array}{c}\text { Turduckening } \\
\text { radius }\end{array}$ & \multicolumn{3}{|c}{ Resolution } \\
& $r_{t}$ & $6 \Delta$ & $4 \Delta$ & $3 \Delta$ \\
\hline small & $0.10 M$ & 11 & 17 & 23 \\
medium & $0.15 M$ & 9 & 14 & 19 \\
large & $0.20 M$ & 7 & 11 & 15
\end{tabular}

Table I: Number of grid points between the turduckening region and radius of the initial apparent horizon $\left(r_{h o r}=0.3758 M\right)$, for three turduckening radii $r_{t}$ and three grid resolutions $d x$. The resolution $d x$ is given in multiples of $\Delta=0.04 M$. If there are too few grid points between the horizon and the turduckening region, information about the turduckening procedure escapes out of the black hole, which must be avoided.

smoothness of the resulting field $A$. If $n=2, A$ will be only $C^{0}$. Increasing $n$ by 2 results in one additional derivative being continuous, so that for $n=4, A$ is $C^{1}$, and for $n=6$, the resulting $A$ is $C^{2}$. Since we need to take two derivatives of the metric, we choose $n=6$ to keep all derivatives continuous. We will show later that using $n=2$ still works, but leads to large errors.

Equation (40) is linear, and we employ a standard conjugate gradient method [44] to solve it. This numerical scheme is rather easy to implement, is robust, and it converges reasonably quickly at the resolutions within reach on current supercomputing hardware. The algorithm has been implemented in the Cactus thorn NoExcision that we have made freely available. ${ }^{4}$

Higher values of $n$ lead to smoother initial data. It is also possible to choose a non-zero right hand side in (40), modifying the shape of the solution in the excised region. In this paper we do not take advantage of this additional freedom. Other turduckening procedures may also be possible and could be directly integrated into the initial data solvers.

\section{B. Distorted rotating black hole evolutions}

We now turn to evolutions of single distorted, rotating black holes and investigate the effects of black hole turduckening on the numerical solution and the extracted waveforms. We use single puncture initial data with a Bowen-York extrinsic curvature [45]. We use a puncture mass $m_{p}=$ 0.751744 and a moderately large angular momentum parameter $S=0.7$, resulting in a black hole with irreducible mass $M_{\text {irr }} \approx 0.925785$, dimensionless spin $a / M=S / M^{2}=0.7$, and mass $M=1.0$ (see e.g. Eq. (27) in [46] for a definition of the horizon mass used here). The ADM mass of the spacetime is $M_{\mathrm{ADM}} \approx 1.00252$.

We chose puncture initial data to be able to compare turducken and puncture evolutions (see also Sec. IV]). We chose a non-zero angular momentum to arrive at a more interesting, nonspherically-symmetric case which also includes gravitational wave emission, as the conformally flat rotating single punctures do not represent the Kerr spacetime [47].

We perform simulations of four different initial data setups. The first is a pure puncture setup where the turduckening procedure is not applied. In the other three cases, the puncture data are modified inside of different turduckening radii: small $\left(r_{t}=0.1 M\right)$, medium $\left(r_{t}=0.15 M\right)$, and large $\left(r_{t}=0.2 M\right)$, but kept unchanged everywhere else. The radius of the initial apparent horizon is $r_{\text {hor }}=0.3758 M$. For all four cases we perform simulations at three different resolutions: low $(d x=$ $0.024 M)$, medium $(d x=0.016 M)$ and high $(d x=0.012 M)$, as measured on the finest refinement level. Table \lists the approximate number of grid points between the horizon and the turduckening region in each of these cases. These runs were performed using the McLachlan code (see Sec. III),

\footnotetext{
4 The Cactus thorn can be obtained via the command svn checkout https://svn.aei.mpg.de:/numrel/AEIThorns/NoExcision/trunk NoExcision
} 

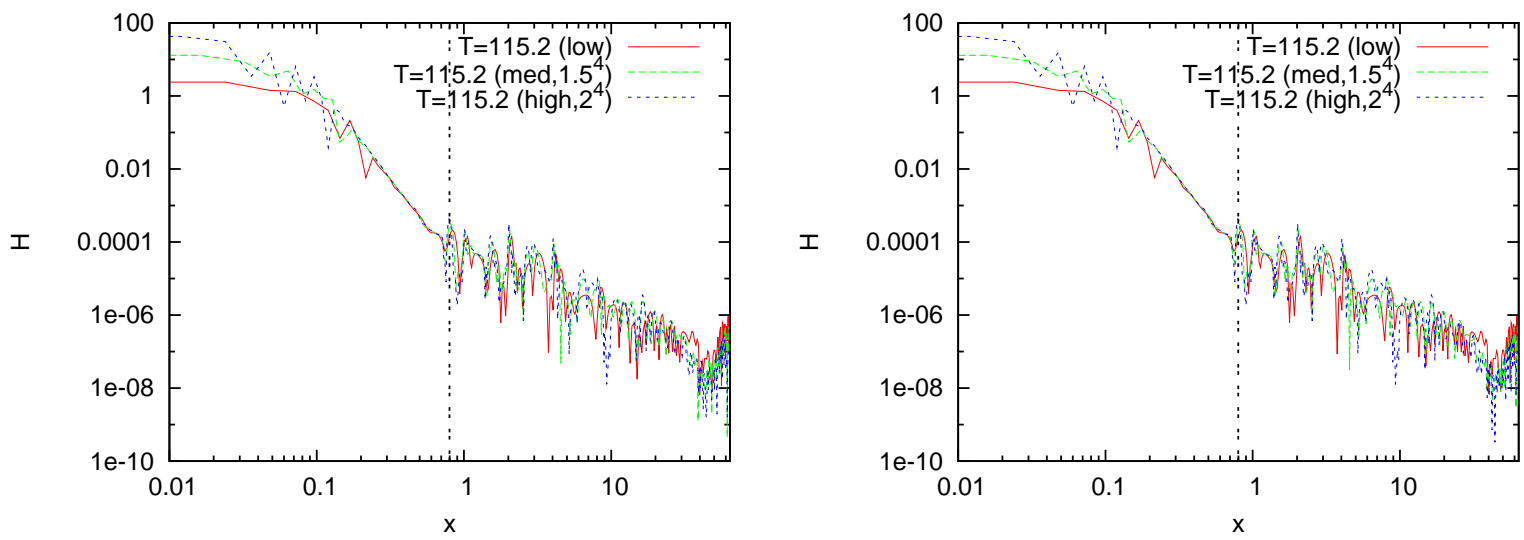

Figure 8: Convergence plot for the Hamiltonian constraint along the $x$ axis at $T=115.2 M$ for the small turduckening region $r_{t}=0.1 M$ (left plot) and for the puncture run (right plot). The medium and high resolution curves have been scaled for 4 th order convergence. The vertical line shows the location of the horizon. The plots cover the range over which the waveforms were extracted (up to $R=60 M$ ). At this time this region is still causally disconnected from the outer boundary.

eight levels of mesh refinement, and the outer boundaries located at $R=256 \mathrm{M}$. Refinement boundaries were placed at $R=[128,64,16,8,4,2,1] M$. Fifth order Kreiss-Oliger dissipation [41] was applied to all evolved variables.

We choose the initial lapse profile as

$$
\alpha_{\text {init }}=\frac{1}{1+m_{p} /(2 r)}
$$

which corresponds to the average of the isotropic lapse and unit lapse. Except for the puncture case, the lapse is further modified in the turduckening region by smoothing it using Eq. (40) with $n=6$. The shift and its time derivative are initially set to zero.

\section{Numerical results}

\section{Constraints}

Figure 8 shows the convergence behaviour for the Hamiltonian constraint for the small turduckening radius $r_{t}=0.1 M$ and the puncture run along the $x$ axis at a late time $T=115.2 M$ (any late time could have been chosen, since the numerical spacetime has essentially become stationary). The vertical line shows the location of the horizon at this time. Similar results hold for the momentum constraint. These results show that at late times the constraints converge to zero at a fourth order rate, except for a few grid points near the origin. Since the stationary solution we are approaching has a $1 / \sqrt{r}$ singularity in the conformal factor [12, 13], this is to be expected, as a neighborhood of the origin is under-resolved. Note also that the convergence plots are practically identical for the turducken and puncture simulations. These results are consistent with the 1D results of Section IV.

\section{Lapse}

The behavior of the lapse is also consistent with the spherically symmetric simulations of Section [V] Figure 9 shows the lapse profile along the $x$ axis at four different times $(T=0 M$, 

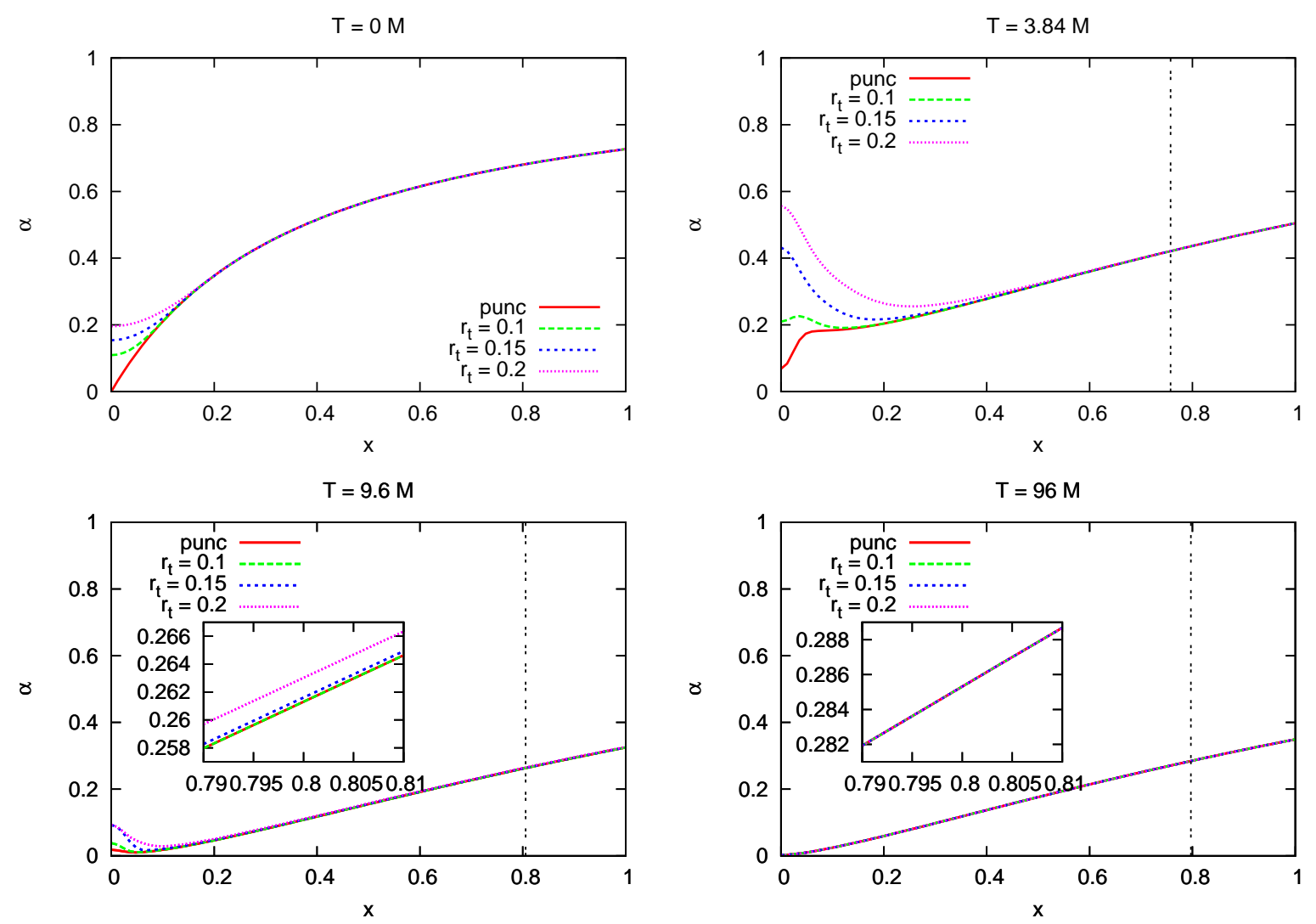

Figure 9: Lapse profile along the $x$ axis for the distorted single black hole evolutions at the times $T=0 M$ (top left), $T=3.84 M$ (top right), $T=9.6 M$ (bottom left), and $T=96 M$ (bottom right) for the high resolution, comparing different turduckening radii. The vertical lines show the location of the apparent horizon. The insets in the bottom graphs enlarge a small region near the horizon. While the lapse profiles differ near the origin, they are very similar at the horizon, and their difference decreases with time. However, for the medium and large turduckening radii, a superluminal gauge mode is clearly escaping. On the other hand the differences between the lapse profiles of the puncture and small turduckening radius cases converge to zero with resolution.

$T=3.84 M, T=9.6 M$, and $T=96 M)$ for the high resolution case. The region with significant differences in the slicing is at all times safely contained within the horizon. However, as can be seen from the inset in the bottom left graph, there are real (but small) differences in the lapse function also outside the horizon at early times. These differences do not converge away with resolution. In other words, the details of the turduckening procedure result in real (but small) differences in the slicing outside of the horizon. With time, the differences become smaller, and are no longer visible in the bottom right graph at $T=96 M$.

\section{Waveforms: convergence with resolution}

We extract the $\ell=2, m=0$ mode of the Weyl scalar $\Psi_{4}$ on coordinate spheres at four different radii $(R=30 M, R=40 M, R=50 M$, and $R=60 M)$, choosing the commonly used hypersurfaceadapted tetrad described e.g. in [48]. Figure 10 shows convergence tests for the waveforms obtained with different turducken radii and with puncture evolutions, in all cases extracted at $R=30 M$.

The top left graph shows the waveform with small turducken radius $r_{t}=0.1 M$ at three different resolutions. The top right graph shows the differences between the different resolutions, scaled for 

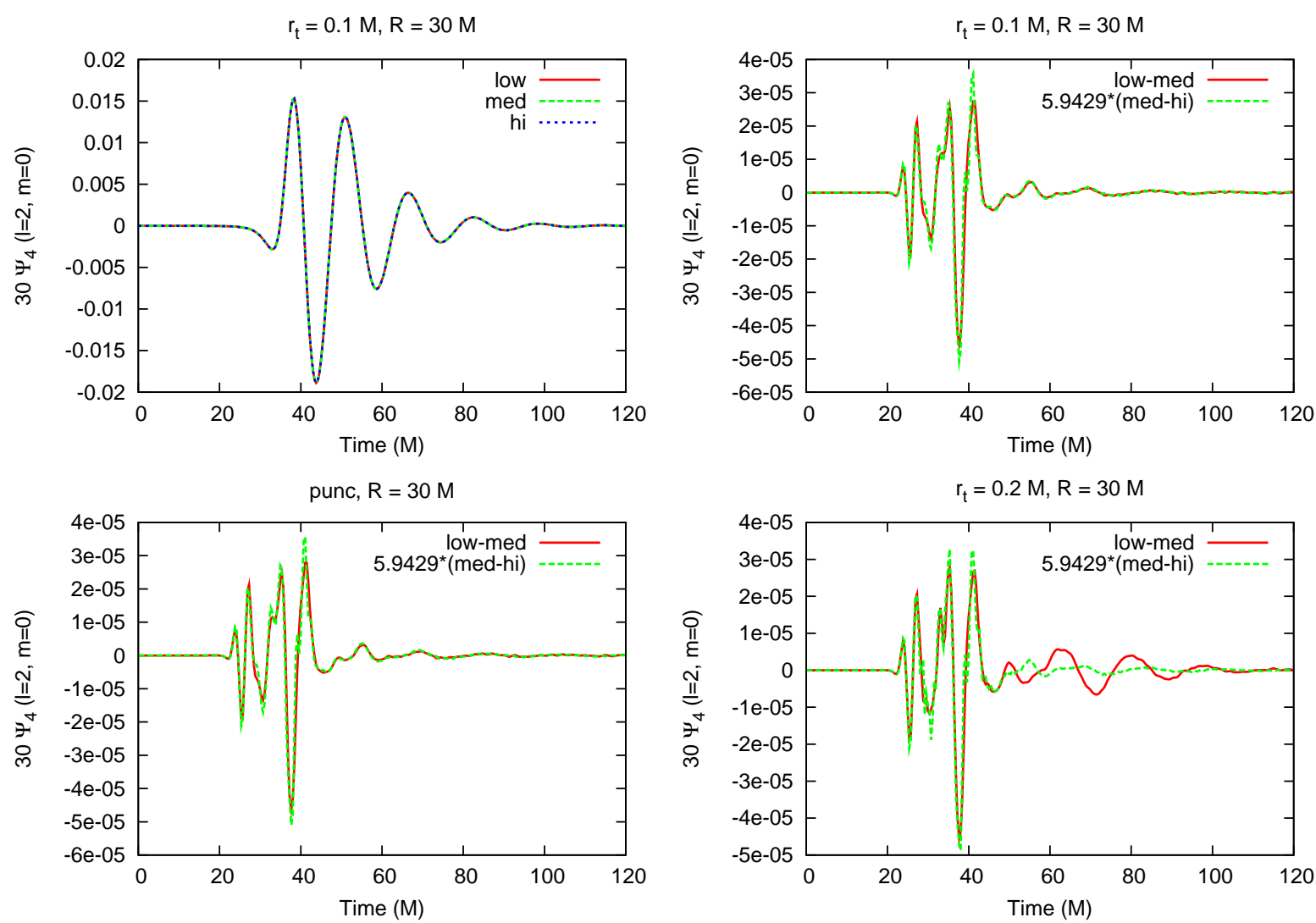

Figure 10: Effect of the turduckening radius on the $\ell=2, m=0$ mode of gravitational waveforms $r \Psi_{4}$, extracted at $R=30 \mathrm{M}$. The top left graph compares all three resolutions, and the top right graph shows their differences, scaled for 4th order convergence, both for the small turduckening region $r_{t}=0.1 M$. The bottom left graph shows the scaled differences for a pure puncture run, and the fact that it looks virtually identical indicates that $r_{t}=0.1 M$ is a good choice for these resolution. On the other hand, the bottom right graph shows the same for the large turduckening radius $r=0.2 M$ where noticeable differences are visible. We attribute this to the smaller number of grid points between the turduckening region and the initial apparent horizon (see table \), so this case is not yet in the convergent regime.

fourth order convergence. The fact that the curves look so similar is a manifestation of clean fourth order convergence.

The bottom left graph shows the scaled differences for a pure puncture run, also indicating clean fourth order convergence. The case $r_{t}=0.15 M$ (not shown here) behaves similarly.

However, the scaled differences for $r_{t}=0.2 M$ (bottom right graph) looks noticeably different. We attribute this to the smaller number of grid points between the turduckening region and the initial apparent horizon (see table【), so that, in the low resolution case, there are too few gridpoints between the turduckening region and the horizon for the numerical waveforms to be effectively isolated from the stuffing. The curve for the difference between the medium and high resolution waveforms is very similar to the other cases, which indicates that it is only the low resolution run that is not in the convergent regime. We expect, though we have not yet confirmed this with numerical experiments, that a convergence test with a higher minimal resolution will also show a lack of convergence if the turduckening region is chosen so that $r_{t}$ is only $\sim 7$ (or fewer) gridpoints away from the horizon at the minimal resolution. Thus, as a rule of thumb, we expect that one should always choose the turduckening region so that $r_{t}$ is more than $\sim 7$ gridpoints away from the horizon. Finite differencing schemes that differ from the one used in our code would probably 

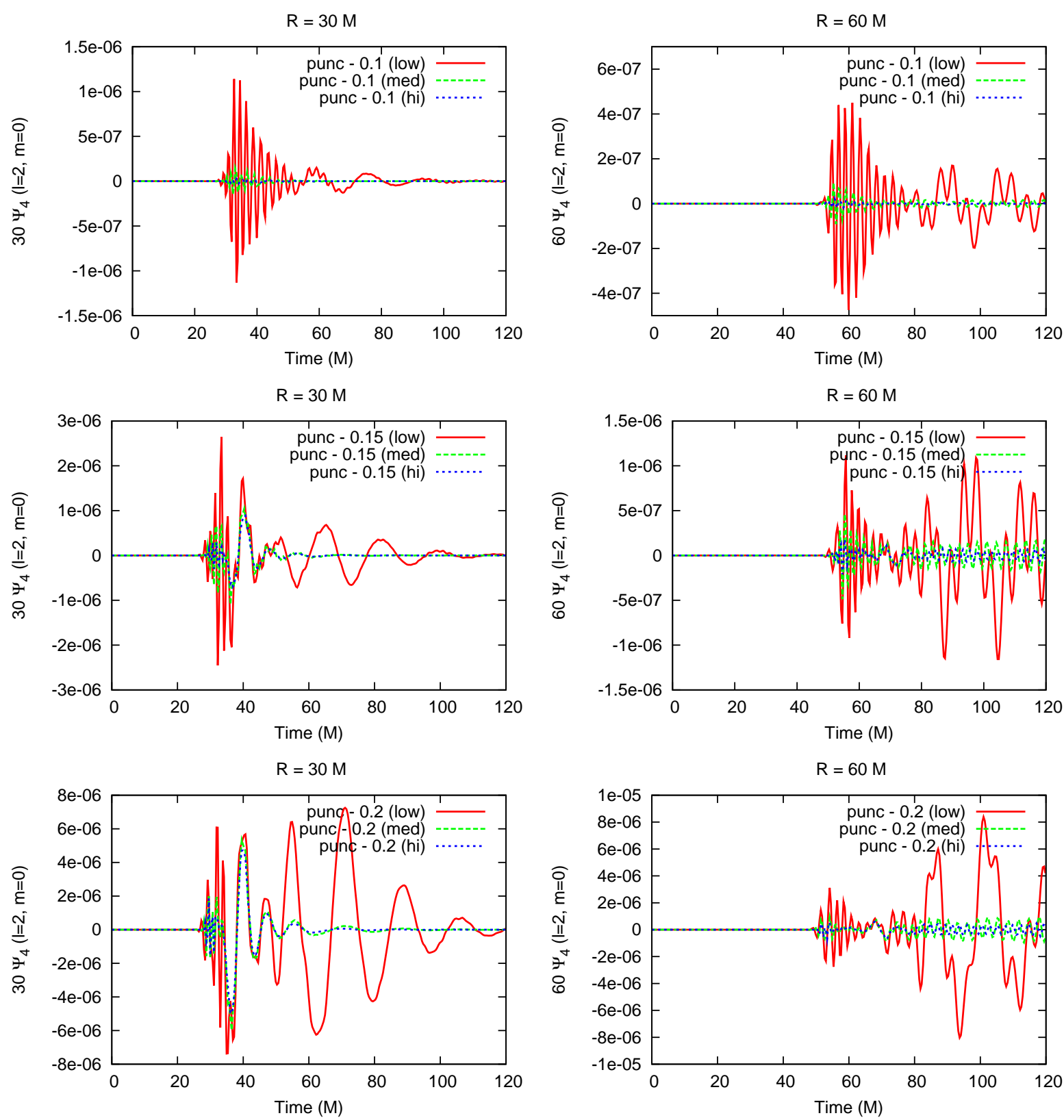

Figure 11: Difference between the $\ell=2, m=0$ mode of $r \Psi_{4}$ for a puncture evolution and for three different turduckening radii, extracted at $R=30 M$ (left-hand graphs) and $R=60 M$ (right-hand graphs). See the main text for the discussion.

require different limits. Experimentation would be necessary on a case-by-case basis.

\section{Waveforms: comparison among different solution methods}

Figure 11 shows, for the three resolutions, the differences between the puncture waveform and the turducken waveforms with turduckening radii $r_{t}=0.1 M, r_{t}=0.15 M$, and $r_{t}=0.2 M$. In all cases two extraction radii are used: $R=30 M$ and $R=60 M$. As can be seen from the figure, the difference between the puncture waveform and the $r_{t}=0.1$ one goes to zero as the resolution is 
increased. This is not true for the $r_{t}=0.15$ and $r_{t}=0.2$ cases, where the difference, especially clear at $R=30 \mathrm{M}$, does not converge to zero with increasing resolution. Differences in the slicing do appear to affect the extracted gravitational wave signal. However, at the larger extraction radius $R=60 M$, the non-convergent part of the waveform difference is significantly smaller.

As already discussed, there are gauge modes that travel at superluminal speeds with our parameter choices and differences in gauge are able to propagate beyond the horizon. This is true independently of whether the initial data are pure puncture or turduckened data. The fact that we only find differences between the puncture waveforms and turducken ones with larger $r_{t}$ does not mean that we consider the puncture waveforms and the turducken waveforms with small $r_{t}$ to be correct, and any deviation from them to be an error. As described above, we modify the initial lapse profile away from the puncture profile only inside of the turduckening region. We would expect that a pure puncture run with a different initial lapse profile (for example, constant lapse equal to one) would also yield significant differences in the lapse at the location of the detector. Instead, the conclusion is that since wave extraction is carried out at a finite radius, our resolutions are high enough so that the seemingly small differences in slicing lead to noticeable differences in waveforms. Furthermore, even though this is a rotating black hole, which cannot be compared directly to a Schwarzschild black hole, we believe that this is the same effect as seen in the 1D spherically symmetric case (see Fig. [6 and its discussion in section IVB). Namely, differences in slicing get trapped inside the black hole for sufficiently small turducken radius (while the puncture evolution can be considered as the limit $r_{t} \rightarrow 0$ ). Note also that the slicing differences (and consequently the waveform differences) are transient since both puncture and turducken runs approach the same trumpet slice at late times.

What we have seen is that, through a proper convergence test, we can detect differences in the waveforms due to different slicings at fixed, finite extraction radii. However, it is far from clear that these differences are of any practical importance. For example, for each of our turduckening radii, the largest difference (at the highest resolution) between a turducken waveform and the puncture one is $6 \times 10^{-6}$. The amplitude of the wave is about 0.015 , so the relative difference is about $4 \times 10^{-4}$.

Finally, we performed experiments at low $(d x=0.024 M)$ and medium $(d x=0.016 M)$ resolutions with a turduckening radius $r_{t}=0.1$, but with $n=2$ in equation (40), so that the evolution fields are only $C^{0}$ at the boundary of the turduckening region. In Figure 12 the left graph shows the difference between the waveforms from runs with $n=2$ and $n=6$ while the right graph shows the difference between the $n=2$ and puncture waveforms. These two graphs are almost identical and a comparison with the top left graph in Figure 11 explains why. As can be seen, the difference between the puncture waveforms and the $r_{t}=0.1$ waveforms is about 10 times larger for $n=2$ than $n=6$. Note that in the $r_{t}=0.1 M$ case the turduckening region is far enough inside the black hole that we do not see gauge differences, so this must be caused by increased numerical noise coming from the less smooth data in the $n=2$ case.

\section{FINAL REMARKS}

In this paper we have analyzed in detail several aspects of the turduckening technique for evolving black holes.

First we presented a detailed analytical study of the constraints propagation for a rather general family of BSSN-type formulations of the Einstein equations. We could appropriately identify a sub-

family for which the constraints propagate within the light cone and give a rigorous justification of the turduckening procedure. At the same time, we showed that in other subfamilies the constraint violations do move superluminally. As a consequence, in those cases, smoothing the interior of 

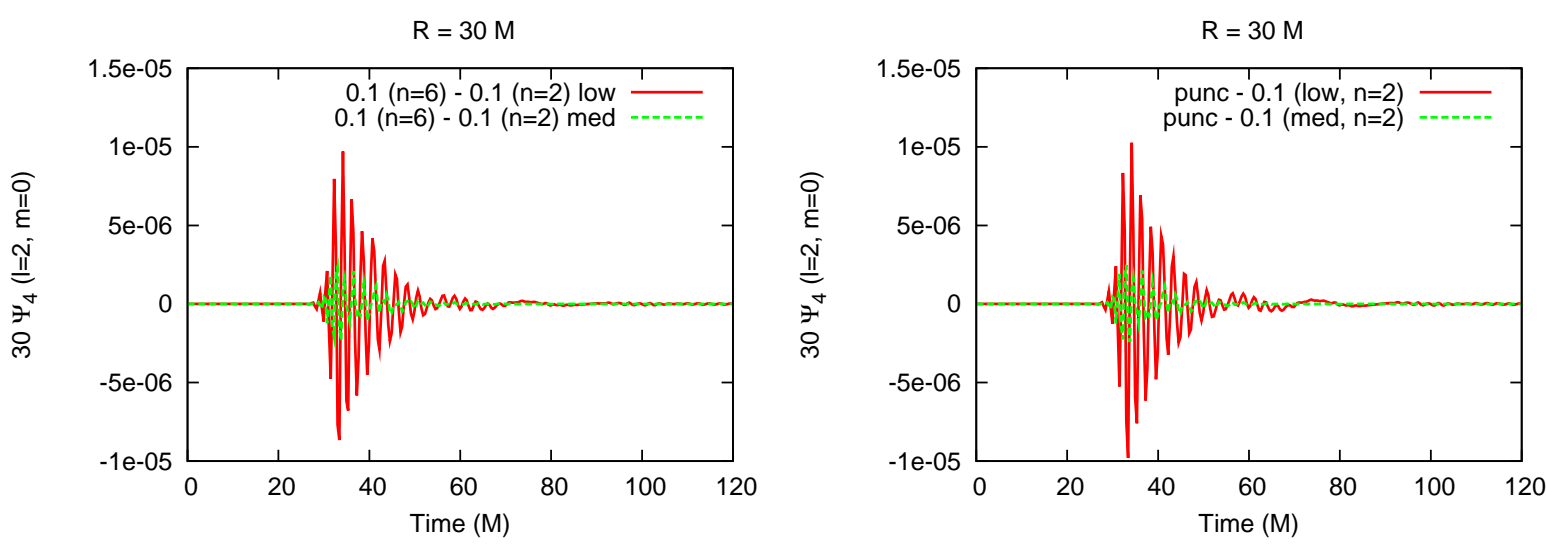

Figure 12: Difference between the $\ell=2, m=0$ mode of $r \Psi_{4}$ for the turducken runs with $n=6$ and $n=2$ (both with $r_{t}=0.1$ ) extracted at $R=30 M$ (left plot). The right plots shows the same for the puncture run and the turducken with $r_{t}=0.1$ and $n=2$.

black holes will result in constraint violations that propagate to the outside.

Through high-resolution spherically symmetric numerical simulations we analyzed in detail the behavior of the constraints and gauge modes. In particular, we confirmed to very high accuracy the predictions of our analytical study. We found that the numerical constraints are preserved outside the black hole when the theory predicts so, and are violated otherwise. We also found cases in which gauge modes do propagate superluminally, escaping from the black hole, and cases in which they are trapped in the inside. These differences in gauge modes have consequences for wave extraction, as discussed below. We also observed that, when the constraints are guaranteed to propagate within the light cone, the region of constraint violations inside the black hole shrinks with time, and that the same final stationary configuration seems to be approached, regardless of the details of the turduckening procedure. We also provided explanations for these features.

Finally, we presented detailed three-dimensional simulations of single distorted black holes, comparing turduckened and puncture evolutions. We studied the effect that these different methods have on the coordinate conditions, constraint violations, and extracted gravitational waves. We found the waves to agree up to small but non-vanishing differences. Our convergence tests showed that those differences are not numerical artifacts but true features of the solution, caused by superluminal gauge modes escaping from the black hole. We also found that these differences in waveforms decay with increasing extraction radius.

\section{Acknowledgments}

O.S. wishes to thank Darío Nuñez for help in deriving the characteristic speeds of the constraint propagation system, P.D. and E.S. thank Christian D. Ott and Jian Tao for help with the McLachlan BSSN code. We also wish to thank Ian Hawke and Denis Pollney for many discussions which ultimately led to the turduckening procedure. Our numerical calculations used the Cactus framework 36, 37] with a number of locally developed thorns, J. Thornburg's apparent horizon finder [49, 50], the GNU Scientific Library [51], and the LAPACK [52] and BLAS [53] libraries from the Netlib Repository [54]. This research was supported in part by NSF PIF Grant 0701566 XiRel [55], and NSF SDCI Grant No. 0721915 Alpaca [56] to Louisiana State University, NSF Grant No. PHY-0600402 to North Carolina State University, Grant CIC 4.19 to Universidad Michoacana, PROMEP UMICH-PTC-195 from SEP Mexico, CONACyT Grant No. 61173, NSF Grant No. 
0801213 to the University of Maryland, and the TeraGrid allocation TG-MCA02N014 to Louisiana State University. We used the supercomputing resources Peyote at the AEI, Santaka at LSU, Eric, Queen Bee, and Tezpur at LONI, and Abe and Tungsten at the NCSA. We also employed the resources of the Center for Computation \& Technology at Louisiana State University, which is supported by funding from the Louisiana legislature's Information Technology Initiative.

[1] D. Brown, O. Sarbach, E. Schnetter, M. Tiglio, P. Diener, I. Hawke, and D. Pollney, Excision without excision, Phys. Rev. D 76, 081503(R) (2007), arXiv:0707.3101 [gr-qc].

[2] C. Bona, T. Ledvinka, C. Palenzuela-Luque, J. A. Pons, and M. Záček, Gauge pathologies in singularityavoidant spacetime foliations (2004), gr-qc/0410079.

[3] A. Arbona, C. Bona, J. Carot, L. Mas, J. Massó, and J. Stela, Stuffed black holes, Phys. Rev. D 57, 2397 (1998).

[4] A. Arbona, C. Bona, J. Massó, and J. Stela, Robust evolution system for numerical relativity, Phys. Rev. D 60, 104014 (1999), gr-qc/9902053.

[5] C. W. Misner, Magic matter, the computational aether, and miner's canary (2001), URL https://drum.umd.edu/dspace/handle/1903/4361

[6] Z. B. Etienne, J. A. Faber, Y. T. Liu, S. L. Shapiro, and T. W. Baumgarte, Filling the holes: Evolving excised binary black hole initial data with puncture techniques, arXiv:0707.2083 [gr-qc].

[7] W. G. Unruh, personal communication to Jonathan Thornburg (1984).

[8] J. Thornburg, Coordinates and boundary conditions for the general relativistic initial data problem, Class. Quantum Grav. 4, 1119 (1987), URL http://stacks.iop.org/0264-9381/4/1119

[9] M. Campanelli, C. O. Lousto, P. Marronetti, and Y. Zlochower, Accurate evolutions of orbiting blackhole binaries without excision, Phys. Rev. Lett. 96, 111101 (2006), gr-qc/0511048.

[10] J. G. Baker, J. Centrella, D.-I. Choi, M. Koppitz, and J. van Meter, Gravitational wave extraction from an inspiraling configuration of merging black holes, Phys. Rev. Lett. 96, 111102 (2006), gr-qc/0511103.

[11] M. Hannam, S. Husa, D. Pollney, B. Brugmann, and N. O'Murchadha, Geometry and Regularity of Moving Punctures, Phys. Rev. Lett. 99, 241102 (2007), gr-qc/0606099.

[12] J. D. Brown, Puncture evolution of Schwarzschild black holes (2007), arXiv:0705.1359 [gr-qc].

[13] M. Hannam, S. Husa, F. Ohme, B. Brügmann, and N. Ó. Murchadha, Wormholes and trumpets: the Schwarzschild spacetime for the moving-puncture generation (2008), arXiv:0804.0628.

[14] H. Beyer and O. Sarbach, On the well posedness of the Baumgarte-Shapiro- Shibata-Nakamura formulation of Einstein's field equations, Phys. Rev. D 70, 104004 (2004), gr-qc/0406003.

[15] C. Bona, J. Massó, E. Seidel, and J. Stela, New Formalism for Numerical Relativity, Phys. Rev. Lett. 75, 600 (1995), gr-qc/9412071.

[16] M. Alcubierre, B. Brügmann, P. Diener, M. Koppitz, D. Pollney, E. Seidel, and R. Takahashi, Gauge conditions for long-term numerical black hole evolutions without excision, Phys. Rev. D 67, 084023 (2003), gr-qc/0206072.

[17] J. R. van Meter, J. G. Baker, M. Koppitz, and D.-I. Choi, How to move a black hole without excision: gauge conditions for the numerical evolution of a moving puncture, Phys. Rev. D 73, 124011 (2006), gr-qc/0605030.

[18] M. Alcubierre, G. Allen, B. Brügmann, E. Seidel, and W.-M. Suen, Towards an understanding of the stability properties of the $3+1$ evolution equations in general relativity, Phys. Rev. D 62, 124011 (2000), gr-qc/9908079.

[19] O. Reula, Hyperbolic methods for Einstein's equations, Living Rev. Relativity 1, 3 (1998), URL http://www.livingreviews.org/lrr-1998-3.

[20] O. Sarbach, G. Calabrese, J. Pullin, and M. Tiglio, Hyperbolicity of the BSSN system of Einstein evolution equations, Phys. Rev. D 66, 064002 (2002), gr-qc/0205064.

[21] H. O. Kreiss and O. E. Ortiz, Some mathematical and numerical questions connected with first and second order time dependent systems of partial differential equations, Lect. Notes Phys. 604, 359 (2002), gr-qc/0106085.

[22] G. Nagy, O. E. Ortiz, and O. A. Reula, Strongly hyperbolic second order Einstein's evolution equations, Phys. Rev. D 70, 044012 (2004), gr-qc/0402123. 
[23] C. Gundlach and J. Martin-Garcia, Symmetric hyperbolic form of systems of second-order evolution equations subject to constraints, Phys. Rev. D 70, 044031 (2004), gr-qc/0402079.

[24] C. Gundlach and J. M. Martin-Garcia, Symmetric hyperbolicity and consistent boundary conditions for second-order Einstein equations, Phys. Rev. D 70, 044032 (2004), gr-qc/0403019.

[25] C. Gundlach and J. M. Martin-Garcia, Hyperbolicity of second-order in space systems of evolution equations, Class. Quantum Grav. 23, S387 (2006), gr-qc/0506037.

[26] O. Sarbach and M. Tiglio, Exploiting gauge and constraint freedom in hyperbolic formulations of Einstein's equations, Phys. Rev. D 66, 064023 (2002), gr-qc/0205086.

[27] G. Calabrese, J. Pullin, O. Reula, O. Sarbach, and M. Tiglio, Well posed constraint-preserving boundary conditions for the linearized Einstein equations, Comm. Math. Phys. 240, 377 (2003), gr-qc/0209017.

[28] O. A. Reula, Strongly hyperbolic systems in general relativity, Journal of Hyperbolic Differential Equations 1, 251 (2004), gr-qc/0403007.

[29] M. E. Taylor, Partial Differenaitl Equations I. Basic Theory (Springer-Verlag, New York, 1996).

[30] J. D. Brown, BSSN in spherical symmetry (2007), arXiv:0705.3845 [gr-qc].

[31] J. D. Brown, Conformal invariance and the conformal-traceless decomposition of the gravitational field, Phys. Rev. D 71, 104011 (2005), gr-qc/0501092.

[32] M. Alcubierre, B. Brügmann, T. Dramlitsch, J. A. Font, P. Papadopoulos, E. Seidel, N. Stergioulas, and $\mathrm{R}$. Takahashi, Towards a stable numerical evolution of strongly gravitating systems in general relativity: The conformal treatments, Phys. Rev. D 62, 044034 (2000), gr-qc/0003071.

[33] C. Lechner, D. Alic, and S. Husa, From tensor equations to numerical code - computer algebra tools for numerical relativity, in SYNASC 2004 - 6th International Symposium on Symbolic and Numeric Algorithms for Scientific Computing, Timisoara, Romania (2004), cs.SC/0411063, URL http://arxiv.org/abs/cs.SC/0411063.

[34] S. Husa, I. Hinder, and C. Lechner, Kranc: a Mathematica application to generate numerical codes for tensorial evolution equations, Comput. Phys. Comm. 174, 983 (2006), gr-qc/0404023.

[35] Kranc: Automated Code Generation, URL http://numrel.aei.mpg.de/Research/Kranc/

[36] T. Goodale, G. Allen, G. Lanfermann, J. Massó, T. Radke, E. Seidel, and J. Shalf, The Cactus framework and toolkit: Design and applications, in Vector and Parallel Processing - VECPAR'2002, 5th International Conference, Lecture Notes in Computer Science (Springer, Berlin, 2003).

[37] Cactus Computational Toolkit home page, URL http://www.cactuscode.org/.

[38] E. Schnetter, S. H. Hawley, and I. Hawke, Evolutions in 3D numerical relativity using fixed mesh refinement, Class. Quantum Grav. 21, 1465 (2004), gr-qc/0310042.

[39] Mesh refinement with Carpet, URL http://www.carpetcode.org/.

[40] L. Lehner, S. L. Liebling, and O. Reula, AMR, stability and higher accuracy, Class. Quantum Grav. 23, S421 (2006), gr-qc/0510111.

[41] H. O. Kreiss and J. Oliger, Methods for the approximate solution of time dependent problems (GARP publication series No. 10, Geneva, 1973).

[42] M. Hannam, S. Husa, N. Ó Murchadha, B. Brügmann, J. A. González, and U. Sperhake, Where do moving punctures go?, Journal of Physics: Conference series (2007), in press, gr-qc/0612097.

[43] T. W. Baumgarte and S. G. Naculich, Analytical representation of a black hole puncture solution, Phys. Rev. D 75, 067502 (2007).

[44] J. R. Shewchuk, An introduction to the conjugate gradient method without the agonizing pain, Tech. Rep., School of Computer Science Carnegie Mellon University Pittsburgh, PA 15213 (1994), URL www.cs.cmu.edu/ quake-papers/painless-conjugate-gradient.pdf.

[45] M. Ansorg, B. Brügmann, and W. Tichy, A single-domain spectral method for black hole puncture data, Phys. Rev. D 70, 064011 (2004), gr-qc/0404056.

[46] O. Dreyer, B. Krishnan, D. Shoemaker, and E. Schnetter, Introduction to Isolated Horizons in Numerical Relativity, Phys. Rev. D 67, 024018 (2003), gr-qc/0206008, URL http://link.aps.org/abstract/PRD/v67/e024018.

[47] J. A. Valiente Kroon, Nonexistence of conformally flat slices in Kerr and other stationary spacetimes, Phys. Rev. Lett. 92, 041101 (2004), gr-qc/0310048.

[48] D. Pollney, C. Reisswig, L. Rezzolla, B. Szilagyi, M. Ansorg, B. Deris, P. Diener, E. N. Dorband, M. Koppitz, A. Nagar, et al., Recoil velocities from equal-mass binary black-hole mergers: a systematic investigation of spin-orbit aligned configurations, Phys. Rev. D 76, 124002 (2007), arXiv:0707.2559 [gr-qc]. 
[49] J. Thornburg, Finding apparent horizons in numerical relativity, Phys. Rev. D 54, 4899 (1996), grqc/9508014.

[50] J. Thornburg, A fast apparent-horizon finder for 3-dimensional Cartesian grids in numerical relativity, Class. Quantum Grav. 21, 743 (2004), gr-qc/0306056, URL http://stacks.iop.org/0264-9381/21/743

[51] GSL: the GNU Scientific Library, URL http://www.gnu.org/software/gsl/.

[52] LAPACK: Linear Algebra Package, URL http://www.netlib.org/lapack/.

[53] BLAS: Basic Linear Algebra Subroutines, URL http://www.netlib.org/blas/.

[54] Netlib Repository, URL http://www.netlib.org/.

[55] XiRel: Next Generation Infrastructure for Numerical Relativity, URL http://www.cct.lsu.edu/xirel/.

[56] Alpaca: Tools for Application-Level Profiling and Correctness Analysis, URL http://www.cactuscode.org/Development/alpaca/ 